\title{
Tissue mimetic hyaluronan bioink containing collagen fibers with controlled orientation modulating cell morphology and alignment.
}

Andrea Schwab ${ }^{1}$, Christophe Helary ${ }^{2}$, Geoff Richards ${ }^{1}$, Mauro Alini ${ }^{1}$, David Eglin ${ }^{1}$, Matteo D'Este ${ }^{1 *}$

Author affiliations and contact information

${ }^{1}$ AO Research Institute, Clavadelerstarsse 8, 7270 Davos, Switzerland

${ }^{2}$ Sorbonne Université | UPMC · Laboratoire de Chimie de la Matière Condensée de Paris (LCMCP), Paris, France

${ }^{*}$ Corresponding author:

Matteo D'Este

matteo.deste@aofoundation.org

Clavadelerstarsse 8, 7270 Davos, Switzerland 


\section{Abstract}

2 Biofabrication is providing scientists and clinicians the ability to produce engineered tissues

3 with desired shapes, and gradients of composition and biological cues. Typical resolutions

4 achieved with extrusion-based bioprinting are at the macroscopic level. However, for

5 capturing the fibrillar nature of the extracellular matrix (ECM), it is necessary to arrange ECM

6 components at smaller scales, down to the micron and the molecular level.

7 In this study, we introduce a bioink containing hyaluronan (HA) as tyramine derivative (THA)

8 and collagen type 1 (Col 1). Similarly to other connective tissues, in this bioink Col is present

9 in fibrillar form and HA as viscoelastic space filler. THA was enzymatically crosslinked under

10 mild conditions allowing simultaneous Col fibrillogenesis, thus achieving a homogeneous

11 distribution of Col fibrils within the viscoelastic HA-based matrix. THA-Col composite

12 displayed synergistic properties in terms of storage modulus and shear-thinning, translating

13 into good printability.

14 Shear-induced alignment of the Col fibrils along the printing direction was achieved and quantified via immunofluorescence and second harmonic generation. Cell-free and cell-laden constructs were printed and characterized, analyzing the influence of the controlled microscopic anisotropy on human bone marrow derived mesenchymal stromal cells (hMSC) migration.

THA-Col showed cell instructive properties modulating hMSC adhesion, morphology and sprouting from spheroids stimulated by the presence and the orientation of Col fibers. Actin filament staining showed that hMSCs embedded into aligned constructs displayed increased cytoskeleton alignment along the fibril direction. Based on gene expression of cartilage/bone markers and ECM production, hMSCs embedded into the bioink displayed chondrogenic differentiation comparable to standard pellet culture by means of proteoglycan production 25 (Safranin O staining and proteoglycan quantification).

26 The possibility of printing matrix components with control over microscopic alignment brings 27 biofabrication one step closer to capturing the complexity of native tissues. 
1 Keywords: 3D bioprinting; extracellular matrix; hyaluronan; collagen; cell spheroid;

2 chondrogenesis

3

\section{$4 \quad 1$ Introduction}

5 Biofabrication aims at engineering constructs recapitulating the complexity of mammal

6 tissues concerning cell types, chemical and biological gradients, and multiscale architecture.

7 In extrusion-based 3D printing (3DP), the resolution is determined by the size of the nozzle,

8 printing speed and offset, distance between nozzle and printing surface, with resolution

9 ranging from the $\mathrm{mm}$ down to the micron range [1]. The physico-chemical properties of the

10 biomaterial and bioink directly influence printing outcome and shape fidelity. The ink is most

11 often a viscoelastic shear-thinning hydrogel (precursor) with good elasticity recovery after

12 high shear and rapid gelation after extrusion [2-4]. In 3DP, high resolution must be

13 compromised for cell viability, with typical resolutions in the hundreds of microns range [5].

14 Thus, for standard extrusion-based techniques, which are the most versatile and widespread,

15 only the macroscopic architecture can be deliberately designed.

16 By combining different biomaterial ink compositions, gradients of material composition and

17 cellular distribution can be produced using 3D printing techniques [6-8]. Despite impressive

18 advances, the field still lacks reliable methods to mimic tissue architectures not only by

19 replicating the ECM composition, but also addressing the (macro)molecular organization

20 within the biomaterial ink at (sub-) micron scale lengths, e.g. on fibrillar levels [9]. However, it

21 is well-known how microarchitectural features provide specific and unique properties to

22 natural tissues [10].

23 Col is the most abundant protein in the ECM, where it is found with a hierarchical fibrillar

24 structure ranging from the triple helix at the molecular level, up to the fibrils and fibers on the

25 microscopic level. Col structure, orientation and spatial arrangement are fundamental

26 towards mechanical stability and anisotropic properties of tissues [11]. The network

27 architecture is also crucial to transmit forces to cells via cell-matrix interaction and

28 contributes to the matrix biochemical environment [12, 13]. 
1 To bring the 3DP technology one step closer to native tissue architectures, it is necessary to

2 capture micro- and nanostructures within macroscopically complex scaffold geometries [14].

3 Microarchitectural properties can be introduced with polymer self-assembling, a process

4 where macromolecules arrange into stable non-covalent structures. Col 1 [15], fibrin [12],

5 cellulose [16] and silk fibroin [17] are the most prominent materials self-assembling into

6 fibrous structures.

7 Most studies printing Col 1 biomaterial inks employ soluble (acidic) and/or non-fibrillar Col 1

8 inks or blended neutralized Col 1 (Col 1 fractions). Printing an acidic or neutral Col 1

9 dispersion $(20-40 \mathrm{mg} / \mathrm{ml})$ of swollen Col fibrils with a high viscosity $\left(>10^{3} \mathrm{P} \cdot \mathrm{s}\right.$ at shear rate of

$10 \quad 0.11 / \mathrm{s}$ ) requires subsequent lyophilization and chemical crosslinking for scaffold stabilization

11 [18]. Neutralized non fibrillar Col $1(8 \mathrm{mg} / \mathrm{ml})$ was held at low temperature before extrusion

12 and gelation was induced by printing at $37^{\circ} \mathrm{C}$ [19]. In another study, neutralized Col 1

13 fractions were printed at high concentrations $(20-40 \mathrm{mg} / \mathrm{ml})$ and lower temperature $\left(4-15^{\circ} \mathrm{C}\right)$

14 during extrusion printing and gelled within pre warmed media [20].

15 However, only few studies investigated the formation and presence of Col 1 fibers within the

16 printed hydrogel construct and the influence on cell migration [21-23]. Moreover, cell

17 embedding is limited in the above-mentioned approaches either due to the acidic conditions

18 or the post processing. Another drawback of printing neutralized Col 1 is the phase

19 separation of the self-assembled fibrils from the liquid when extruded [23].

20 Hyaluronic acid or hyaluronan $(\mathrm{HA})$ is another abundant ECM component osmotically

21 capable of holding large amounts of water, and thus functioning as a space filler for fibrillar

22 matrix components, thus providing compressive strength through fluid retention [24]. To our

23 knowledge, methods to control the orientation of Col 1 fibers within a viscoelastic HA-based

24 matrix for bio-fabrication has not been addressed before.

25 The aim of this study was the development of a THA-Col 1 composite biomaterial ink and the 26 workflow to 3D print it, fabricating constructs with homogeneous composition and controlled

27 Col fibril orientation at the microscopic level. In addition, the impact of the microscopic 28 anisotropy on the behavior of the embedded hMSC was analyzed (Fig. 1). To this aim, the 


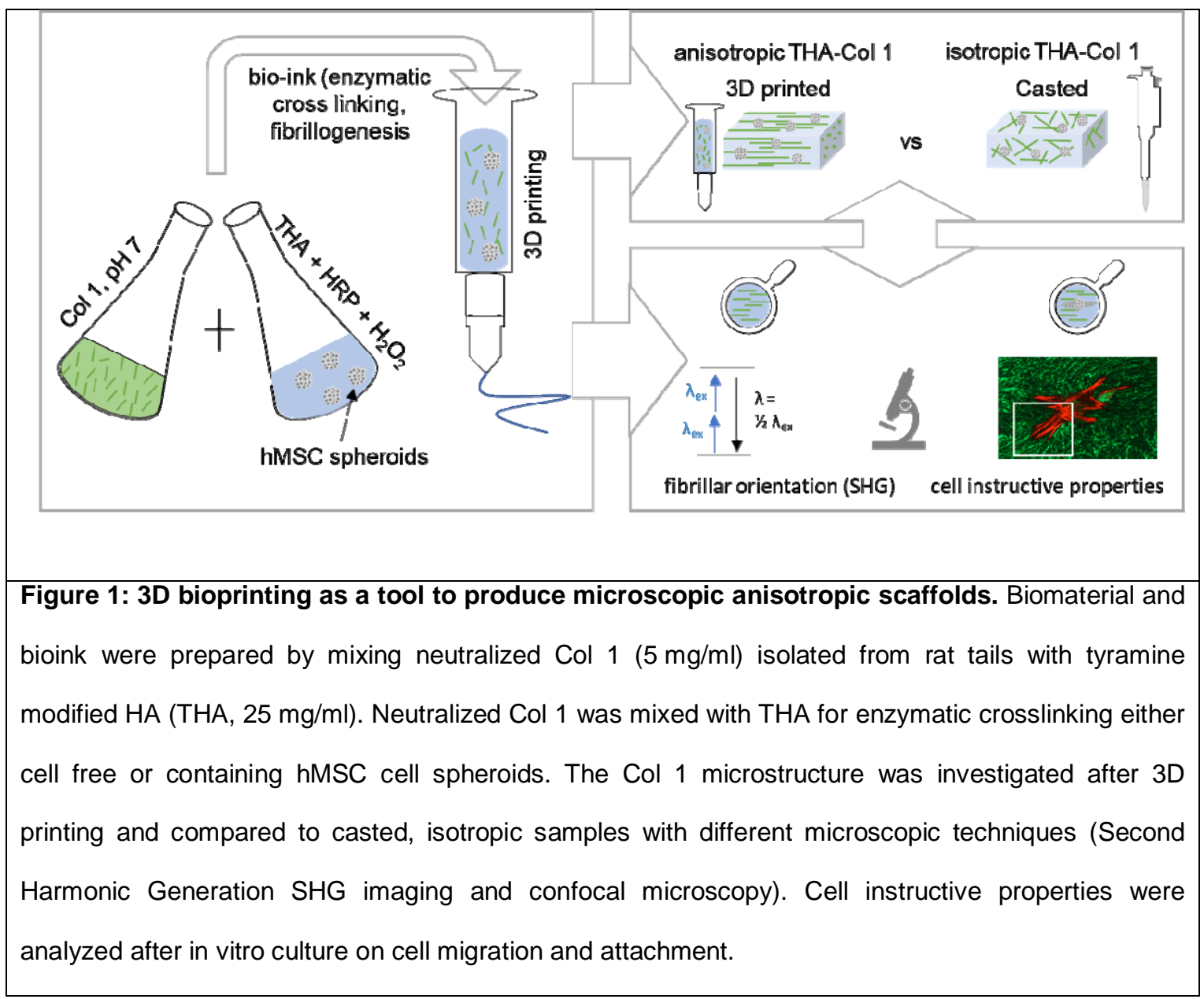

2 tyramine derivative of HA (THA) was employed to form a continuous matrix where solubilized

3 Col 1 was homogeneously dispersed; THA gelation and Col fibrillogenesis occurred without

4 mutual interference. THA was additionally crosslinked with visible light for final shape

5 fixation, thus achieving a uniform THA matrix containing Col fibrils, which were aligned via

6 the shear forces experienced during the extrusion process. Fibril alignment and cytoskeleton

7 orientation were quantified and compared to casted, isotropic constructs. hMSC spheroids

8 were embedded in isotropic constructs with different compositions of THA and Col to

9 compare their sprouting and chondrogenic differentiation. 


\section{Materials and Methods}

\subsection{Tyramine modified hyaluronic acid (THA) synthesis}

3 THA was synthesized as previously described [25]. Briefly, HA (280-290 kDa, $5 \mathrm{mM}$

4 carboxylic groups, Contipro Biotech S.R.O) was functionalized via 4-(4,6-dimethoxy-1,3,55-

5 triazin-2-yl)-4-mehtylmorpholinium chloride (DMTMM, TDI) amidation with tyramine by mixing

6 with a stoichiometric ratio of $1: 1: 1$. Functionalization was performed for $24 \mathrm{~h}$ at $37^{\circ} \mathrm{C}$. THA

7 was precipitated by dropwise adding Ethanol (96\% v/v), isolated with Gooch filter No. 2 and

8 dried. The degree of substitution was $6.6 \%$ as determined by absorbance reading at $275 \mathrm{~nm}$

9 (Multiskan $^{\mathrm{TM}} \mathrm{GO}$ Microplate Spectrophometer, Thermo Fisher Scientific).

\subsection{THA-Col 1 hydrogel preparation}

THA $(25 \mathrm{mg} / \mathrm{ml}$ ) was reconstituted with minimum Essential Medium alpha (a-MEM, Gibco) supplemented with $10 \% \mathrm{v} / \mathrm{v}$ bovine fetal serum containing peroxidase from horseradish (HRP, Sigma Aldrich) at the specified concentration at $4^{\circ} \mathrm{C}$ under agitation. THA for turbidity measurement was reconstituted with PBS. Enzymatic crosslinking was initiated by adding hydrogen peroxide $\left(\mathrm{H}_{2} \mathrm{O}_{2}\right.$, Sigma Aldrich) in different concentrations and incubated for $30 \mathrm{~min}$ at $37^{\circ} \mathrm{C}$. THA-Col 1 composite was prepared by neutralizing Col $1(5 \mathrm{mg} / \mathrm{ml}$, Collagen I rat tail in $0.2 \mathrm{~N}$ acetic acid, Corning) with $\mathrm{NaOH}$ and adding to THA before enzymatic crosslinking was initiated. Mixing ratios by volumes of THA $(25 \mathrm{mg} / \mathrm{ml})$ with Col $1(5 \mathrm{mg} / \mathrm{ml})$ are given in brackets for all experimental set ups described in the following paragraphs. For cell encapsulation, hMSC spheroids were resuspended with THA before adding $\mathrm{H}_{2} \mathrm{O}_{2}$. Additional light crosslinking using $0.2 \mathrm{mg} / \mathrm{ml}$ of Eosin $\mathrm{Y}$ as photoinitiator (Sigma Aldrich) was done for all printing experiments to stabilize the printed structures and the cell migration and cell differentiation studies. Non-aligned constructs were produced by pipetting the hydrogel precursor with a positive displacement pipette (CP1000, inner diameter ID $1.0 \mathrm{~mm}$, Gilson)

26 into custom made silicon molds (6 $\mathrm{mm}$ in diameter). Enzymatic crosslinking was performed 27 for all samples for $30 \mathrm{~min}$ at $37^{\circ} \mathrm{C}$. 


\subsection{D bioprinting of THA-Col}

3 An extrusion based bioprinter (3D Discovery ${ }^{\top \mathrm{M}}$, RegenHU) was used to prepare anisotropic

4 hydrogels (0.25" cylindrical needles, 15G: ID $1.36 \mathrm{~mm}, 0.2$ bar, writing speed $8 \mathrm{~mm} / \mathrm{s}$; $18 \mathrm{G}$ :

5 ID $0.84 \mathrm{~mm}, 1.6$ bar, writing speed $8 \mathrm{~mm} / \mathrm{s}$, Nordson EFD) with subsequent light crosslinking

$6 \quad(515 \mathrm{~nm}$ LED, speed $4 \mathrm{~mm} / \mathrm{s})$. Biomaterial and bioink were transferred into 3CC barred (ID

$72.3 \mathrm{~mm}$, Nordson) for enzymatic crosslinking $\left(30 \mathrm{~min}, 37^{\circ} \mathrm{C}\right)$, printed with the above-

8 mentioned parameters and transferred into culture media (cell embedded hydrogel) or PBS

9 (cell free).

10 A complex microstructure to mimic microarchitecture of Col fibers in articular cartilage was

11 accomplished with CAD Software (RegenHU). Fibrillar structure in the superficial zone (SZ at

12 the surface) was realized by printing 3 layers of parallel lines, whereas the Middle (MZ) and

13 deep zone (DZ) were designed as circular structures to mimic the arch like geometry.

\subsection{Rheological characterization}

Viscoelastic properties of the biomaterial inks were analyzed using Anton-Paar MCR-302

17 rheometer with $1^{\circ}$ cone-plate geometry and gap distance of $0.049 \mathrm{~mm}$ at $20^{\circ} \mathrm{C}$. Silicone oil (Sigma Aldrich) was applied to the external border to prevent drying during the measurement. Viscosity was measured with shear rate ranging from $0.011 / \mathrm{s}$ to $1001 / \mathrm{s}$ ( $n=2 /$ group) to evaluate shear thinning behavior of the enzymatically crosslinked THA (0.3 U/mI HRP, $\left.0.52 \mathrm{mM} \mathrm{H}_{2} \mathrm{O}_{2}\right)$, THA-Col $1(1: 1 \mathrm{v} / \mathrm{v})$ and Col 1 in a rotational experiment.

Oscillatory tests (amplitude sweep: frequency $1 \mathrm{~Hz}$, amplitude $0.01-100 \%$ strain; frequency sweep: amplitude $1 \%$ strain, frequency $0.01-100 \mathrm{~Hz}, \mathrm{n}=2$ /group) were performed with parallel plate measuring system at $20^{\circ} \mathrm{C}$ to characterize the elastic modulus of THA and 


\subsection{Turbidity measurement}

2 Fibrillation of Col 1 was evaluated by absorbance reading at $313 \mathrm{~nm}$ at $37^{\circ} \mathrm{C}$ at 5,30 and 360 min after induction of neutralization of Col 1 and enzymatic gelation ( $n=2-5$

4 samples/group) of freshly prepared materials with a Multiskan ${ }^{\mathrm{TM}} \mathrm{GO}$ Microplate Spectrometer

5 (Thermo Scientific). The following solutions were analyzed: neutralized Col 1, acidic Col 1, 6 THA $\left(0.075 \mathrm{U} / \mathrm{ml} \mathrm{HRP}, 0.26 \mathrm{mM} \mathrm{H}_{2} \mathrm{O}_{2}\right)$ and THA-Col $1(1: 1$ and 1:2 v/v) composite 7 undergoing enzymatic cross-linking during measurement of Col 1 fibrillogenesis.

8 Temperature was held at $37^{\circ} \mathrm{C}$ to induce enzymatic crosslinking of THA and stabilize Col 1 9 fibrillation.

\subsection{Second harmonic generation (SHG) imaging}

12 To visualize Col 1 fibers $\left(0.3 \mathrm{U} / \mathrm{ml} \mathrm{HRP}, 0.52 \mathrm{mM} \mathrm{H}_{2} \mathrm{O}_{2}\right)(1: 1 \mathrm{v} / \mathrm{v}) \mathrm{SHG}$ images were acquired 13 with a MaiTai multi-photon laser equipped confocal microscope (Leica SP8). SHG signal was collected with HyD detector between $437-453 \mathrm{~nm}\left(\lambda_{\mathrm{ex}} 880 \mathrm{~nm}\right.$, output power ap $\left.1.7 \mathrm{~W}\right)$. Additionally, transmitted light and autofluorescence signal $(510-600 \mathrm{~nm})$ was acquired with varying emission wavelength for SHG detector ( $\lambda_{\mathrm{em}} 420-436 \mathrm{~nm}$ and $\left.\lambda_{\mathrm{em}} 454-470 \mathrm{~nm}\right)$ to check for the specificity of SHG signal at $437-453 \mathrm{~nm}$. For deep imaging a long-distance objective (25x water-immersion objective) was used to acquire z-stacks with optimal settings for each sample. Image series acquired with Leica Application Suite X software (LAS X, Leica) were processed with ImageJ (National Institute of health, NIH).

\subsection{Cell culture}

\subsubsection{Cell isolation}

hMSCs were isolated from bone marrow aspirate with full ethical approval (Ethics committee of University of Freiburg Medical Centre - EK-Freiburg: 135/14) as described elsewhere [26]. hMSCs were sub-cultured with a-MEM (Gibco) supplemented with $10 \%$ v/v Sera Plus bovine 
1 Fibroblast Growth Factor (FGFb, Fitzgerald Industries International) in a humidified

2 atmosphere of $5 \% \mathrm{CO}_{2}$ with media change every second day.

3

$4 \quad$ 2.7.2 Cell migration and viability study

5 Cell spheroids were prepared from hMSC (passage 3-4) in ultra-low attachment petri dish

6 (Corning) with a-MEM media supplemented with $10 \%$ bovine serum and $100 \mathrm{U} / \mathrm{ml}$ Penicillin,

$7100 \mathrm{ug} / \mathrm{ml}$ Streptomycin before embedded in hydrogels. Cell spheroid suspension was added

8 for (enzymatic) crosslinking $\left(0.3 \mathrm{U} / \mathrm{ml} \mathrm{HRP}, 0.39 \mathrm{mM} \mathrm{H}_{2} \mathrm{O}_{2}\right)$ into THA-Col 1(1:1 v/v) or THA or

9 Col 1 at final cell density of $3 \mathrm{Mio} / \mathrm{ml}$ and cultured for 8 days. For THA-Col 1 and THA, an additional light crosslinking of printed and casted samples was done at $515 \mathrm{~nm}$.

11 To evaluate cell migration, samples at day 0,3 , and 8 were stained with phalloidin as described in 2.8.1 and fluorescent images were taken for subsequent quantification of migration length and area ( $n=3-5$ spheroids/timepoint) described in Chapter 2.9. Live and Dead assay was performed during the migration experiment of hMSC spheroids embedded in THA-Col $1\left(0.3 \mathrm{U} / \mathrm{ml} \mathrm{HRP}, 0.39 \mathrm{mM} \mathrm{H}_{2} \mathrm{O}_{2}, 1: 1 \mathrm{v} / \mathrm{v}\right)$ of $3 \mathrm{D}$ printed and casted samples at day 1 and day $6 . \mathrm{H}_{2} \mathrm{O}_{2}$ concentration was selected in a range known not to be cell toxic [27]. After washing with PBS, samples were incubated with $2 \mu \mathrm{M}$ Calcein AM (Sigma Aldrich) and $1 \mu \mathrm{M}$ Ethidium homodimer-1 (Sigma Aldrich) for $30 \mathrm{~min}$ at $37^{\circ} \mathrm{C}$, washed with PBS and imaged using confocal microscope (LSM800, Carl Zeiss). Dead cells were stained with Ethidium homodimer-1 in red $\left(\lambda_{\mathrm{ex}} 561 \mathrm{~nm}\right)$, cytoplasm of living cells was stained with Calcein-AM in green $\left(\lambda_{\mathrm{ex}} 488 \mathrm{~nm}\right)$.

\subsubsection{Chondrogenic differentiation}

For chondrogenic differentiation, hMSC (passage 2) were seeded in 6 well microwell plate (AggreWellTM 400 plate) at 1.67 Mio/well for 3 days to from cell spheroids. Chondrogenic media was composed of high glucose Dulbecco's Modified Eagle Medium (DMEM HG,

27 Gibco) supplemented with non-essential amino acids (1\% v/v, Gibco), ascorbic acid 2 
1 (1\% v/v, Corning), TGF $\beta 1$ (10 ng/ml, Fitzgerald) and antibiotics (10 U/ml Penicillin, $10 \mathrm{ug} / \mathrm{ml}$

2 Streptomycin, Gibco). Cell spheroids were embedded in THA-Col 1 (0.5 U/ml HRP, $0.65 \mathrm{mM}$

$3 \mathrm{H}_{2} \mathrm{O}_{2}, 5 \% \mathrm{w} / \mathrm{v}$ Col $\left.1,1: 1 \mathrm{v} / \mathrm{v}\right)$ at final cell concentration of $5 \mathrm{Mio} / \mathrm{ml}$ hydrogel volume

4 (400,000 cells/scaffold) before enzymatic gelation, additionally light cross linked, and

5 cultured for 21 days with chondrogenic media. Media was changed 3 times a week. For

6 positive control, standard hMSC pellets were prepared by seeding 250,000 hMSCs into each

7 of a 96 V-Bottom well plate (Ratiolab) and cultured under same conditions.

\section{$9 \quad 2.8$ Histological processing and staining}

$10 \quad$ 2.8.1 Actin filament-Col 1 immunofluorescence staining

11 For actin filament staining in combination with Col 1 immuno fluorescent staining, printed and 12 casted THA-Col $1(1: 1 \mathrm{v} / \mathrm{v})$ were fixed with $4 \%$ neutral buffered formalin (Formafix AG) at 13 room temperature and stored in PBS at $4^{\circ} \mathrm{C}$ upon staining.

14 Cytoskeletal organization of hMSC sprouting from cell spheroids embedded in enzymatically 15 cross linked THA-Col 1 samples $\left(0.3 \mathrm{U} / \mathrm{ml} \mathrm{HRP,} 0.39 \mathrm{mM} \mathrm{H}_{2} \mathrm{O}_{2}\right)$ were analyzed on day 0 and 16 after 6 days culture with Phalloidin staining to visualize actin filaments. Hydrogels were 17 permeabilized with $0.5 \% \mathrm{v} / \mathrm{v}$ Triton $\mathrm{X}-100$ (Sigma Aldrich) for $10 \mathrm{~min}$ at room temperature, blocked with $10 \%$ bovine serum (Sera plus) for 30 min and stained with Phalloidin-TRITC (2 $\mu \mathrm{g} / \mathrm{ml}, \mathrm{P} 1951$, Sigma Aldrich) for $45 \mathrm{~min}$ at room temperature. Immunofluorescence staining for Col 1 was processed directly after phalloidin staining by overnight incubation with 21 primary antibody (COL 1, 1:5,000 dilution with PBST, monoclonal, Sigma Aldrich). 22 Secondary antibody Goat anti mouse, Alexa fluor 488 (Invitrogen, 1:600 diluted with PBST) was incubated for $1 \mathrm{~h}$. Cell nuclei were stained with DAPI (2 $\mu \mathrm{g} / \mathrm{ml}$, Sigma Aldrich) for 10 min. Samples were washed between every step with Tween-20 (P1379, Sigma Aldrich) 0.1\% v/v in PBS and stored in PBS for microscopy.

26 A confocal microscope (LSM800, Carl Zeiss) was used to acquire fluorescence images (25x, $2740 x$ water-immersion objectives) to visualize Col 1 matrix and cells. Col 1 fibers within the 28 hydrogel matrix were stained in green $\left(\lambda_{\mathrm{ex}} 488 \mathrm{~nm}\right)$, cell cytoskeleton in red $\left(\lambda_{\mathrm{ex}} 561 \mathrm{~nm}\right)$ and 
1 cell nuclei were stained with DAPI in blue $\left(\lambda_{\mathrm{ex}} 405 \mathrm{~nm}\right)$. For all samples, $\mathrm{z}$-stack images were

2 acquired for the three single channels and processed with ImageJ (National Institute of

3 health, $\mathrm{NIH}$ ) to generate $2 \mathrm{D}$ z-projection (max intensity) images. Brightness and contrast

4 settings were adjusted to increase contrast of the single channel images. Merged images of

5 single channels were created with the image overlay tool (merge channels) with ImageJ

6 software $(\mathrm{NIH})$. Images of Col 1 stained samples and cytoskeleton were further processed

7 with ImageJ to quantify fiber orientation and direction of migration as described in 2.10.

\section{$9 \quad 2.8 .2$ Cryo embedding}

10 For histological staining, samples were fixed for $30 \mathrm{~min}$ with $4 \%$ neutral buffered formalin 11 (Formafix $A G)$ at room temperature and stored, washed with PBS and processed with 12 Sucrose $(150 \mathrm{mg} / \mathrm{ml}$ and $300 \mathrm{mg} / \mathrm{ml}$, Sigma Aldrich) before being embedded in tissue 13 freezing medium (Leica). Samples were cut with a cryostat microtome (HM 500 OM, Zeiss) to $148 \mu \mathrm{m}$ slices and kept at $-20^{\circ} \mathrm{C}$ until processed for histological staining with safranin O-fast 15 green.

\subsubsection{Safranin O-Fast Green staining}

To stain the proteoglycans in ECM within hydrogel samples and cell pellets, safranin $O$ staining was performed after 21 days of chondrogenic differentiation. Slides were washed with water to remove the cryo-compound and stained with Weigert's Haematoxylin (12min, room temperature, Sigma Aldrich), blued with tap water (10 min), rinsed with deionized water and stained with Fast Green $(0.02 \%$ w/v, Fluka) for 6 min to visualize collagenous matrix in green/blue. After washing with acetic acid (1\%, Fluka) samples were incubated with safranin $\mathrm{O}(0.1 \%, 15 \mathrm{~min}$, Polysciences) to stain proteoglycans in red. After washing with deionized water, staining was differentiated with Ethanol (70\%, Alcosuisse) and subsequently dehydrated with series of alcohols (ethanol $96 \%$, ethanol absolute, xylene) and cover slipped (Eukitt, Sigma Aldrich). Microscopic evaluation was performed with brightfield microscope (Olympus BX63, Olympus). 


\section{$2 \quad 2.9$ Quantification of cell migration length and area}

3 A sprout morphology tool in Image $(\mathrm{NIH})$ was used to quantify the migration length on

4 phalloidin stained MAX projection images. For bead and sprout detection the mean threshold

5 method was selected with sample specific adjustment of values (Bead detection: blur radius

6 for bead detection 1.0, minimum bead radius $30-110 \mu \mathrm{m}$, dilate beads by factor 1.0-2.8;

7 Sprout detection: sprout detection radius $1.0 \mathrm{um}$, minimal plexus area $5,000 \mu^{2}$, minimal

8 sprout area $500 \mu \mathrm{m}^{2}$ ). Migration area was calculated based on resulting black and white

9 mask from Sprout morphology tool results with ROI area measurement. The calculated area

10 includes the area of the cell spheroids.

\subsection{Evaluation of Col 1 fiber and cytoskeleton alignment}

2D fast Fourier transform (FFT) of images acquired with SHG and confocal microscopy for

Col 1 immunostaining were used for the evaluation of Col 1 fiber alignment. For both microscopic techniques, single channel images at one focus plane were used $(n=2-3$ samples with 3 focus planes at the beginning, center and end of a printed filament) to investigate the homogeneity of alignment at different z-locations within the strut. For quantification of cytoskeleton orientation, MAX projection images of the red channel $(561 \mathrm{~nm})$ of $n=4$ spheroids at day 6 were processed. The Oval profile plug-in in ImageJ $(\mathrm{NIH})$ was chosen for quantitative evaluation of the Col 1 fiber and cytoskeleton orientation as detailed described elsewhere [28, 29]. In brief, single images were processed with ImageJ $(\mathrm{NIH})$ including unsharp mask, FFT processed, rotated $90^{\circ} \mathrm{C}$ right, a circular selection with radius 512 made, oval plug in for radial sum executed with number of points 360 . The resulting grey values were normalized to the maximum grey values of each single image and mean values including standard deviation for both, SHG and confocal images, were shown in the bar diagram. 


\section{$1 \quad 2.11$ Gene expression analysis: Real-Time Quantitative Polymerase Chain}

2 Reaction (PCR)

Total RNA was isolated from the samples (THA-Col 1, THA-Col 1 MSC and MSC pellet) at day 0,14 and 21 using TriReagent® (Molecular Research Centre Inc.) following the manufacturers' protocol. RNA quantity was measured with NanoDrop 1000 spectrophotometer (Thermo Fischer). cDNA synthesis of $1 \mu \mathrm{g}$ RNA was performed with Vilo Superscript (Invitrogen) according to the manufacturer's protocol. Reverse transcription was carried out with Thermocycler (Mastercycler gradient, Eppendorf) including a pre-heating at $25^{\circ} \mathrm{C}$ for $10 \mathrm{~min}$, followed by $42^{\circ} \mathrm{C}$ for $120 \mathrm{~min}$, inactivation of $\mathrm{RT}$ for $5 \mathrm{~min}$ at $85^{\circ} \mathrm{C}$ and cooling down to $4^{\circ} \mathrm{C}$.

For real time PCR, 10ul of reaction mixture containing TaqMan Universal Master Mix (Thermo Fischer), primer and probes, DEPC water and cDNA was loaded into 384 well plates. PCR was run with an initial heating to $50^{\circ} \mathrm{C}$ for $2 \mathrm{~min}, 95^{\circ} \mathrm{C}$ for $10 \mathrm{~min}, 40$ cycles at $95^{\circ} \mathrm{C}$ for $15 \mathrm{~s}$ with the annealing at $60^{\circ} \mathrm{C}$ for $1 \mathrm{~min}$.

Relative gene expression of the chondrogenic associated genes Aggrecan ( $A C A N$ ), Col type 2 (COL2A1) and SOX9, fibrous- and hypertrophy- associated genes Col type 1 and type $\mathrm{X}$ (COL1A1, COL10A1), RUNX2 as well as the endogenous control ubiquitin $C$ (UBC) were calculated using $2^{-\Delta \Delta C q}$. The $U B C$ housekeeping gene has been proven for stability in the present conditions. Two different controls MSC pellets and MSC embedded in THA-Col 1 at day 0 were used for the respective samples. Details on the primers and probe sequence as well as catalogue numbers of Assay-on-Demand (Applied Biosystems) are listed in supplementary (Tab A1).

\subsection{Quantification of Glycosaminoglycans (GAG) and DNA}

To quantify GAGs and DNA within MSC pellets and cell free and cell laden THA-Col 1 hydrogels ( $n=3$ samples/group and time point), samples were digested with Proteinase $K$ $\left(0.5 \mathrm{mg} / \mathrm{ml}\right.$, Sigma Aldrich) at $56^{\circ} \mathrm{C}$. DNA content was measured in duplicates using Quant$\mathrm{iT}^{\mathrm{TM}}$ PicoGreen (Invitrogen) assay according to the manufacturer's instructions. Fluorescence 
1 was measured with a plate reader (Tecan infinite $200 \mathrm{PRO}$, Tecan) at $485 \mathrm{~nm}$ excitation and

$2535 \mathrm{~nm}$ emission including a DNA standard curve. The amount of proteoglycans was

3 determined in duplicates by dimethylene blue dye method with absorbance measurement at

$4 \quad 535 \mathrm{~nm}$ including a Chondroitin 4-sulfate sodium salt from bovine trachea (Fluka

5 BioChemika) standard [30]. For calculation of GAGs released into media the cell free THA-

6 Col 1 values were subtracted from the results of hMSC embedded in THA-Col 1.

\section{$8 \quad 2.13$ Statistical Analysis}

9 All samples were measured in technical duplicates of $n=2-4$ biological replicates per group 10 and time point and displayed as box plot including mean value or as mean values with 11 standard deviation. Statistical analysis was performed with Graph Pad Prism (Prism 8, USA).

12 Cell migration length and migration area as well as PCR data was analyzed with multiple t13 test. Values of the three sample groups (THA, Col 1 and THA-Col 1) were compared at three 14 time points (day 0 , day 3 and day 8 ) and corrected for multiple comparisons using Holm15 Sidak method. Normalization of GAG to DNA values were evaluated using two-way Anova 16 (comparing means of each sample between day 0 and day 21 and between samples at the 17 two time points) with Sidak post hoc test to correct for multiple comparisons. Statistical significance was assumed for $p$-values $<0.05$.

\section{Results}

\subsection{Morphology and mechanical properties of the composite network.}

21 THA-Col 1 composites were prepared dispersing Col 1 into THA, with fibrillogenesis induced 22 by the $\mathrm{pH}$ rise and THA crosslinking triggered by $\mathrm{H}_{2} \mathrm{O}_{2}$ addition and incubation at $37^{\circ} \mathrm{C}$. The 23 presence of Col 1 fibers in THA-Col 1 composite hydrogels was characterized by three 24 independent techniques: SHG imaging, confocal microscopy, and turbidimetry (Fig. 2). 


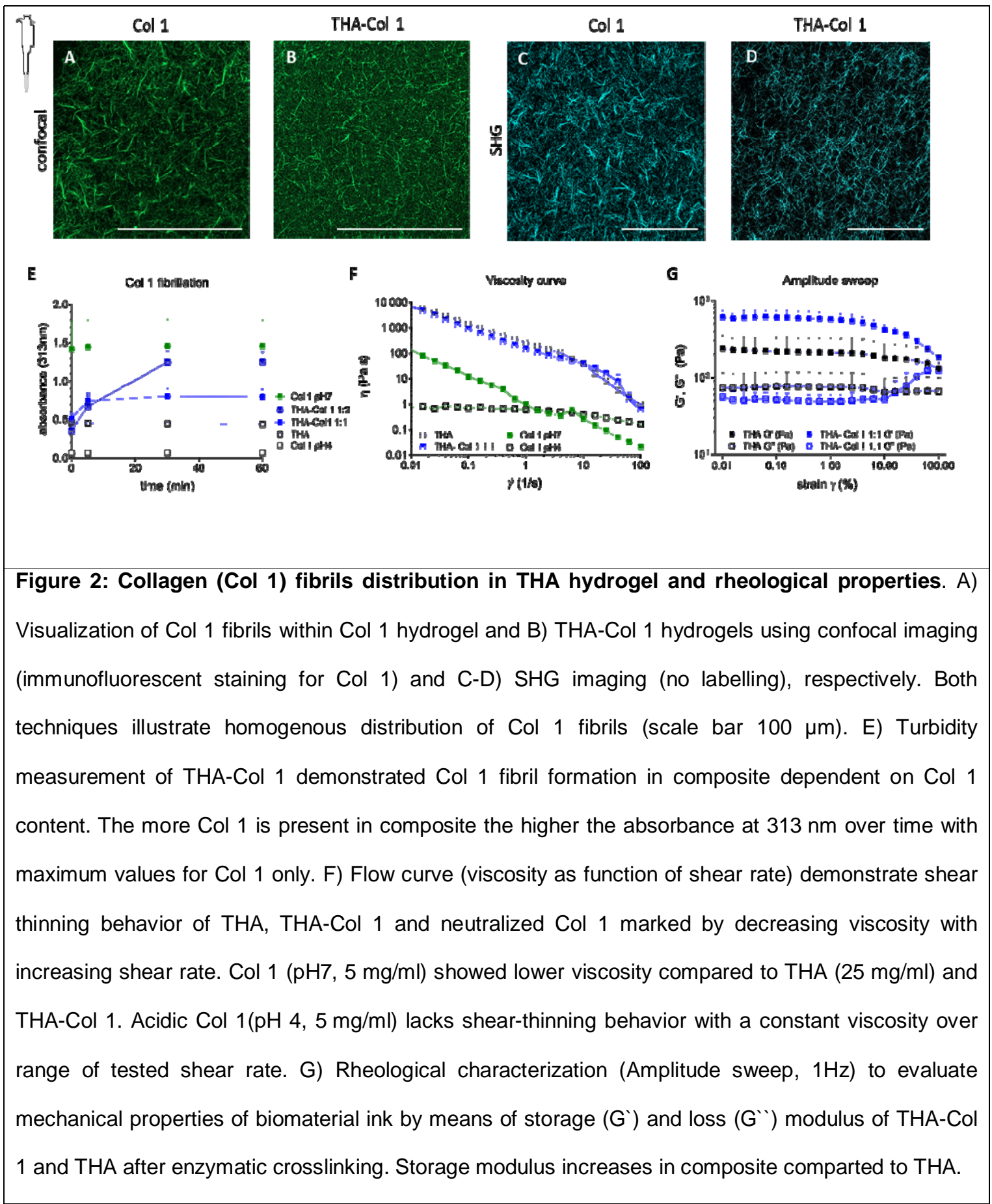

3 As positive control Col 1 hydrogel was included for all measurements. The turbidity

4 measurement allowed evaluating the fibrillogenesis kinetics (Fig. 2 E). An increase in

5 absorbance $(313 \mathrm{~nm}$ ) over time was observed ranging from 0.4 to 0.8 for THA $1: 1 \mathrm{Col} 1$ and

6 from 0.06 to 1.3 for THA 1:2 Col 1, indicative of Col 1 fibrillation within the composite. The 
1 maximum absorbance values increased with the Col 1 content within the materials, with the

2 highest values around 1.5 absorbance units for the neutralized Col 1 positive control, which

3 was fibrillar from the beginning of the measurement. After 30 minutes of incubation at $37^{\circ} \mathrm{C}$ a

4 plateau was reached for all groups. Pure Col 1 in acidic and in neutralized solution, and THA

5 showed constant values in turbidity assay without change in absorbance values over time.

6 The fibrillation of Col 1 was further corroborated by imaging techniques. Both, SHG imaging

7 (Fig. $2 \mathrm{C}$ and D) and confocal microscopy of Col 1 immunofluorescent stained samples (Fig.

$82 \mathrm{~A}$ and $\mathrm{B}$ ), showed obvious presence of Col 1 fibers with a homogenous distribution within

9 THA. Fiber morphology was visibly different, with a thinner and a denser fiber network in

10 THA-Col 1 compared to Col 1 control. Quantification of fibril size and distribution was not

11 possible since the fibrils cross different focal planes resulting in non-reliable values. Images

12 acquired with confocal and SHG microscopy illustrate identical trend in fibrillar

13 characteristics.

$14 \mathrm{HRP}$ concentration, $\mathrm{H}_{2} \mathrm{O}_{2}$ content and mixing ratios of THA and Col 1 were selected based

15 on the requirements for rheological properties and extrudability of the biomaterial ink and Col

161 fibrillogenesis. The viscosity curve clearly demonstrated the shear thinning behavior of the

17 biomaterial ink with an overall higher viscosity of $7 \mathrm{kPa} \cdot \mathrm{s}$ for THA-Col 1 and $6 \mathrm{kPa} \cdot \mathrm{s}$ for THA

18 at $0.011 / \mathrm{s}$, and monotonic decrease for increasing shear rates. At a shear rate higher than

1950 1/s the viscosity of THA-Col 1 fell below the value for THA. Neutralized Col 1 (5 mg/ml)

20 viscosity was overall lower, ranging from $0.13 \mathrm{kPa}$.s at $0.011 / \mathrm{s}$ to $1 \mathrm{~Pa} \cdot \mathrm{s}$ at $11 / \mathrm{s}$. Acidic Col

$211(\mathrm{pH}=4)$ showed an evident reduction in shear-thinning with viscosity ranging from $0.8 \mathrm{~Pa} \cdot \mathrm{s}$

22 to $0.6 \mathrm{~Pa}$.s. Therefore, THA-Col 1 retained the shear thinning behavior required for the

23 biomaterial ink extrusion. An increase in storage modulus was observed in the THA-Col 1

24 composite hydrogel compared to THA (both samples were prepared at the same THA

25 concentration for matching the concentrations of the enzymatic crosslinking agents, Fig. 2

$26 \mathrm{G})$.

27 The amplitude sweep showed similar profile for THA and THA-Col 1, with the Col 1 presence

28 implicating an increase in storage modulus from $220 \mathrm{~Pa}$ to $608 \mathrm{~Pa}$ at $0.1 \%$ strain, but similar 
1 decrease trend at higher strain (Fig. 2 G). This behavior is indicative that THA viscoelastic

2 properties are not disrupted by the formation of the composite with Col 1.

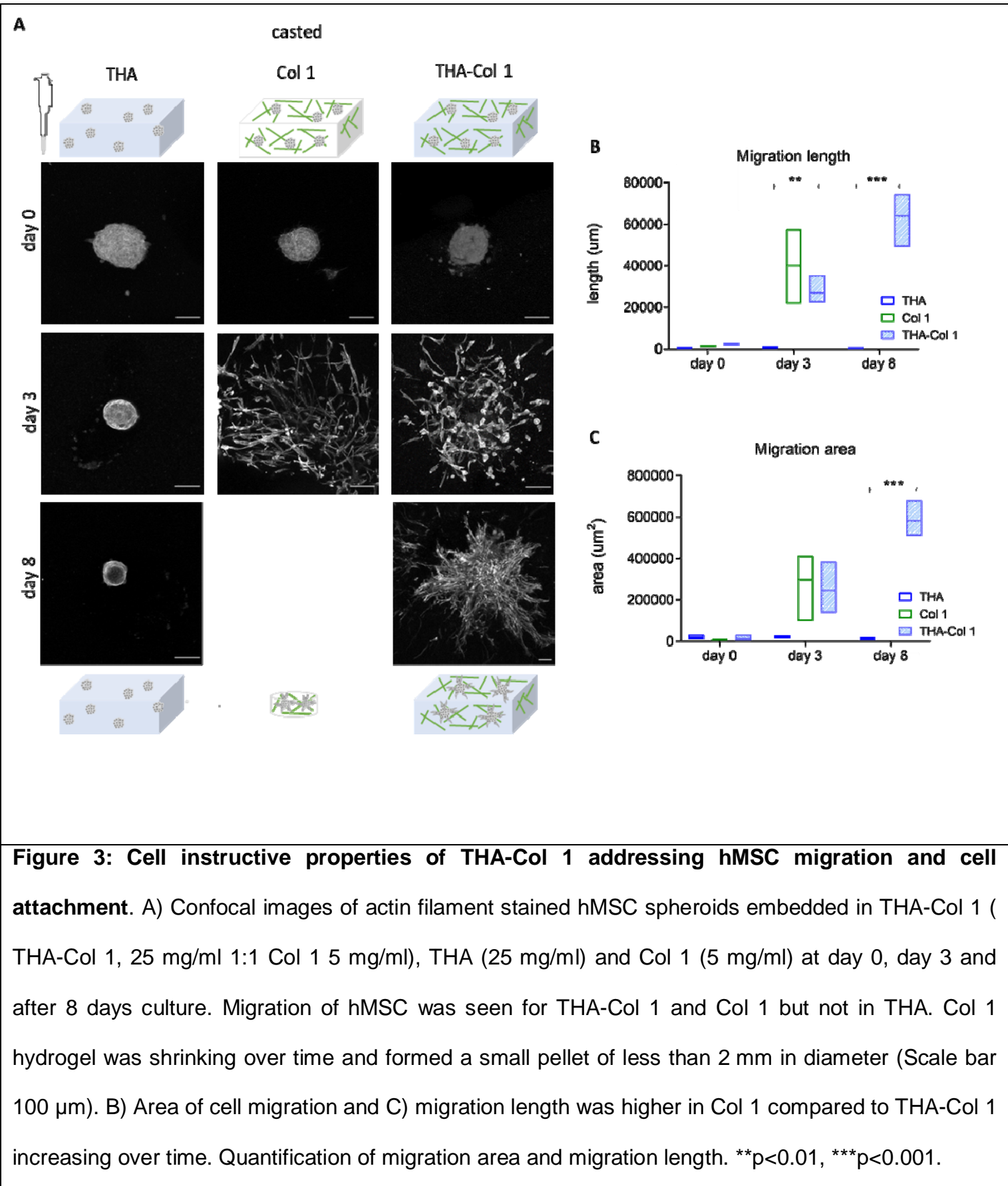

3

43.2 Cell instructive properties of THA-Col 1 bioink

5 hMSCs spheroids were embedded into bioink made of THA-Col 1 and into its single

6 components to determine how the composition influences cell attachment and sprouting from 
1 the spheroids. Fig. 3 A illustrates a panel of MAX projection images stained for actin

2 filaments on day 0 , day 3 and day 8 . Cells migrated out of the uniformly dispersed spheroids

3 into the biomaterials in presence of Col 1 (THA-Col 1 and Col 1), whereas no migration was

4 observed in THA hydrogels.

5 Migration length and migration area were quantified with the ImageJ sprout Morphology Tool

6 on MAX projections images stained with phalloidin. Mean values for both parameters confirm

7 the highest migration in Col 1 (migration length: $40165 \mu \mathrm{m}$; migration area: $297 \mu^{2}$ ),

8 followed by THA-Col 1 (migration length: $26964 \mu \mathrm{m}, \mathrm{p}=0.0050$ compared to THA day 3;

9 migration area: $246 \mu \mathrm{m}^{2}, \mathrm{p}=0.0720$ compared to THA day 3) on day 3 . From day 3 to day 8

10 the migration increased further for $\mathrm{Col} 1$ and THA-Col 1 (THA-Col 1 migration length:

$1163838 \mu \mathrm{m}, \mathrm{p}=0.0005$ compared to THA day 8; migration area: $582 \mu \mathrm{m}^{2}, \mathrm{p}=0.0001$ compared

12 to THA day 8). In THA, migration area and migration length of hMSC spheroids remained

13 unvaried for the whole duration of the experiment. Due to significant shrinkage of Col

141 hydrogels the quantification of the two parameters on day 8 was not possible for for Col 1

15 (Fig. 3 B-C). The differences in spheroid size derived from the variation in focus plane within

16 the 3D constructs. 


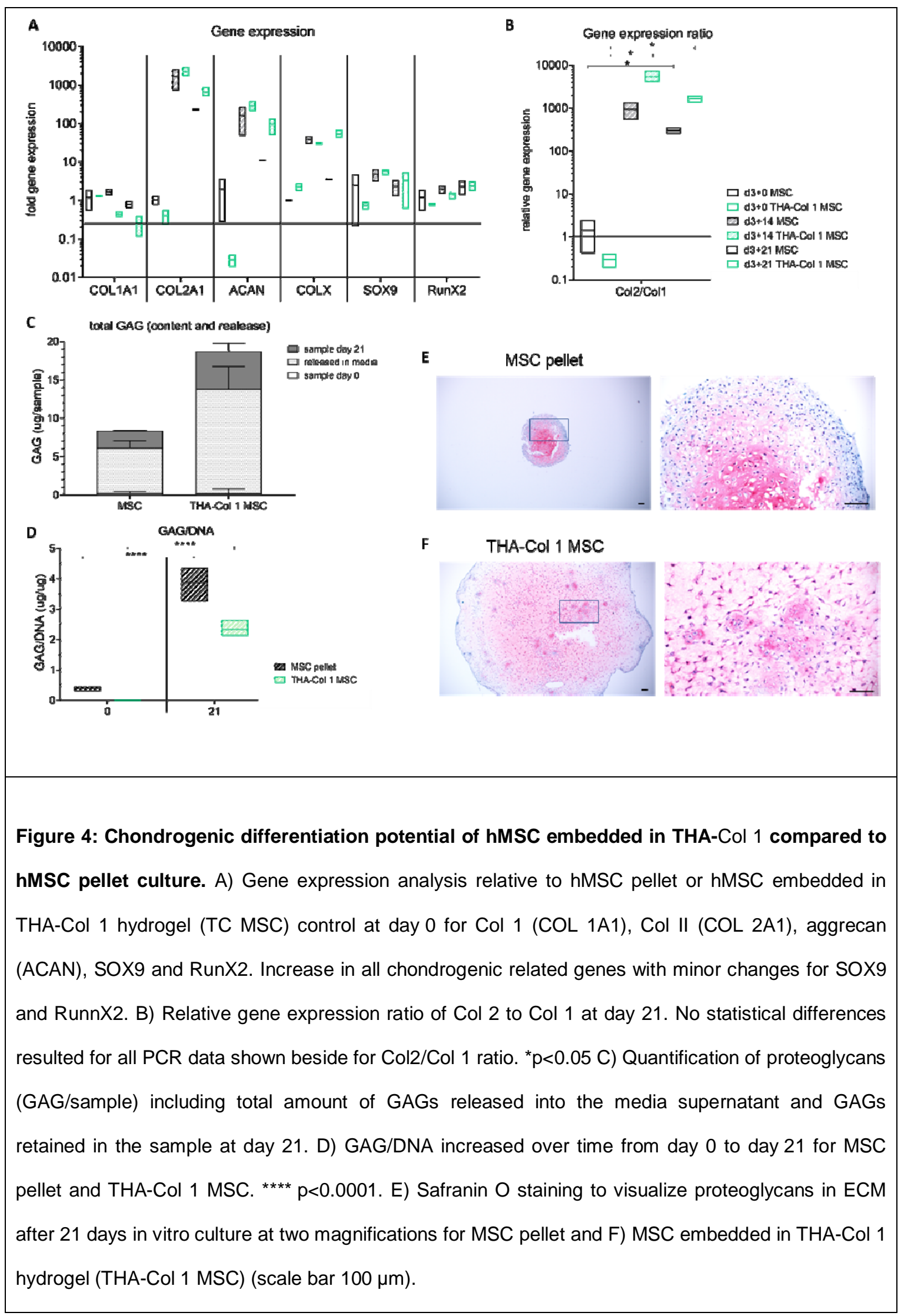




\subsection{In vitro chondrogenic differentiation}

2 Chondrogenic differentiation potential of hMSC spheroids embedded in isotropic THA-Col 1

3 was compared to hMSC pellet culture as positive control, and was evaluated by qPCR,

4 safranin-O staining and total GAG/DNA content. Gene expression analysis of hMSCs

5 spheroid embedded in THA-Col 1 (Fig. 4 A) showed an increase in chondrogenic related

6 markers COL2A1 (1,000-10,000 fold), ACAN (10-1,000 fold) and SOX9 (<20 fold) over time,

7 similarly to hMSCs pellet. COL1A1 was slightly down regulated $(0.1-1.0$-fold), while

8 COL10A1 showed an increase of up to 100 times for the two groups at day 14. At day 21,

9 COL10A1 stayed constant for hMSC embedded THA-Col 1 whereas the fold change relative

10 to control sample decreased to $<10$ for hMSCs pellet. RUNX2 upregulation was much less

11 pronounced (<10-fold change) compared to the extracellular matrix associated genes. The

12 ratio COL2A1/COL1A1 (Fig. $4 \mathrm{~B}$ ), was higher for hMSC spheroids embedded in THA-Col 1

13 (day 14: $5353 \pm 1699, p=0.0242$ compared to day 0 , day $21: 1650 \pm 409, p=0.0293$

14 compared to day 0 ) compared to hMSCs pellet (day 14: $931 \pm 568 p=0.1467$ compared to

15 day 0 , day $21: 300 \pm 69 p=0.0260$ compared to day 0$)$.

16 At day 21, an increase in proteoglycans resulted for both groups (Fig. 4 C-D). Total amount

17 of GAGs increased from $0.46 \pm 0.38 \mathrm{ug} / \mathrm{ml}$ to $4.48 \pm 0.13 \mathrm{ug} / \mathrm{ml}(\mathrm{p}<0.0001)$ for hMSCs pellet and from $1.18 \pm 0.20 \mathrm{ug} / \mathrm{ml}$ to $4.48 \pm 0.69 \mathrm{ug} / \mathrm{ml}(\mathrm{p}<0.0001)$ for hMSCs spheroids embedded

19 in the bioink. Release of proteoglycans in the media was $5.88 \pm 0.92 \mu \mathrm{g}$ and $13.58 \pm 2.93 \mu \mathrm{g}$,

20 respectively. Both groups retained around $25 \%$ of the total produced proteoglycans within the 21 sample (MSC pellet $26.8 \%$, THA-Col 1 MSC 23.3\%). Normalizing total GAG to DNA values 22 in the samples increased for hMSCs pellet within 21 days to $3.88 \pm 0.42(p<0.0001)$ and for hMSCs embedded in THA-Col 1 to $2.34 \pm 0.24$ ( $p<0.0001)$.

24 The histological staining confirmed the chondrogenic differentiation. After 21 days of culture 25 a homogenous distribution of safranin O positive staining was observed for hMSCs pellet and hMSC embedded in THA-Col 1 (Fig. 4 E-F). The hMSC spheroids were no longer visible in

27 the point of initial seeding because of the migration throughout the whole hydrogel, resulting in a homogenous cell distribution. hMSCs morphology was comparable between the cells in 
1 the pellet and the cells embedded in the hydrogel; most cells appeared to be condensed or

2 rounded with a low population of spindle shaped cells at day 21.

3 Overall, this experiment pointed out that hMSC embedded in the isotropic THA-Col 1 bioink

4 is permissive to cell migration, GAG retention and that it retains the same chondrogenic 5 potential as the gold standard pellet culture.

6

\section{$7 \quad 3.4$ 3DP to fabricate microscopically anisotropic scaffolds}

8 After optimizing THA-Col 1 biomaterial ink, the Col 1 presence and fibrillar orientation were

9 characterized. SHG imaging and confocal microscopy were used to visualize the Col 1 fibrils in the THA-Col 1 biomaterial ink after extrusion printing. The fibrillar structure of Col 1 in the composite was preserved after printing and resulted in an anisotropic material. parallel aligned Col 1 fibers along the direction of the printing.

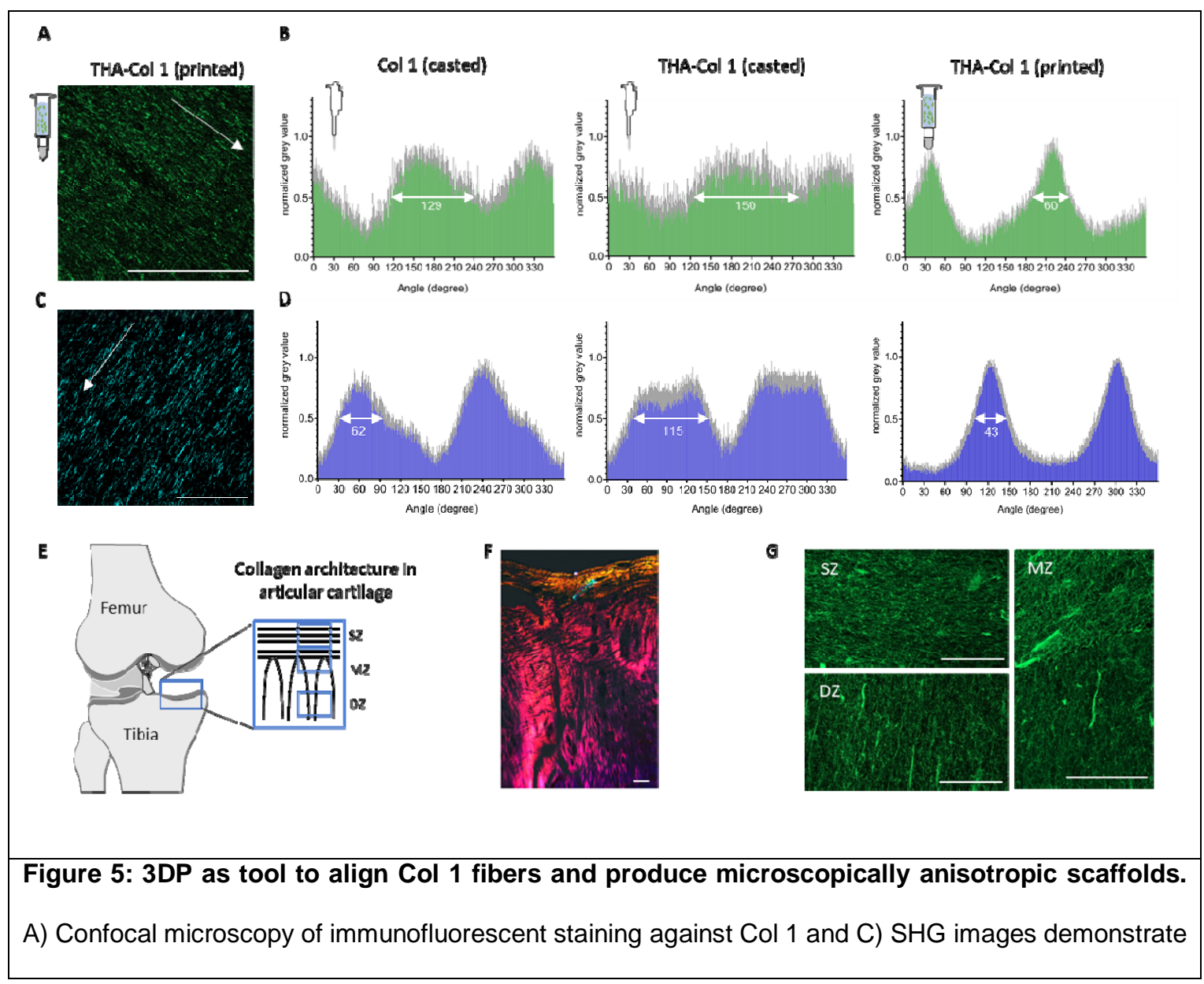


anisotropic fibers in THA-Col 1 biomaterial ink (scale bar $100 \mu \mathrm{m}$ ). Unidirectional orientation of parallel Col 1 fibers aligning along the printing direction (white arrow indicated printing direction). B, D) Bar diagrams (mean values \pm standard deviation) display the quantification of fiber alignment in casted Col 1, casted THA-Col 1 (ID $1.0 \mathrm{~mm}$ ) and printed THA-Col 1 (15G: ID $1.36 \mathrm{~mm}, 0.25$ " cylindrical needle) from images acquired either with confocal microscope (B, green) or SHG (D, blue). Independently of microscopical method the printed THA-Col 1 samples resulted in unidirectional orientation which was more random for casted THA-Col 1 and Col 1 . White arrow in the bar diagram indicate the peak width at a normalized grey value of 0.5 . E) Schematic illustration of Col 1 fiber alignment in articular cartilage. F) Polarized light image of articular cartilage illustrating horizontal fibers in superficial zone (SZ) and vertical orientation in deep zone (DZ) (scale bar $50 \mu \mathrm{m})$. G) Confocal images of immunefluorescent labelled Col 1 mimicking hierarchical fiber orientation in articular cartilage with parallel horizonal fibers in the SZ, more isotropic fibers in the middle zone (MZ) and parallel vertical fibers in the DZ (scale bar $50 \mu \mathrm{m}$ ).

1

2 To further characterize this property, the ImageJ Oval plug in was used to quantify the 3 alignment of THA-Col 1 after printing and compare to casted THA-Col 1 and casted Col 1.

4 Casted THA-Col 1 resulted in the least anisotropic properties, followed by casted Col 1. 5 Comparing the peak width of the preferred orientation in the printed samples at normalized 6 grey value of 0.5 (SHG: 43 degree, confocal microscopy: 60 degree) to the corresponding 7 casted one (SHG: 115 degree, confocal microscopy: 150 degree) and the casted Col 1 8 (SHG: 62 degree, confocal microscopy: 129 degree) a clear narrowing of the Col 1 fiber 9 orientation dispersity was observed for the printed sample (Fig. 5 B and D). The control over the parallel fiber orientation was used to produce a construct imitating the 11 Col orientation in knee articular cartilage. Fibrillar structure in the superficial zone (SZ at the surface) was realized by printing parallel lines, whereas the Middle (MZ) and deep zone (DZ) by an arch like geometry shown in Fig. $5 \mathrm{E}$ and $\mathrm{G}$ with an overall sample size of $1.4 \times 1.6 \mathrm{~cm}$. Immunofluorescent staining against Col 1 clearly shows the fibrillar alignment in the three zones with horizontal fibers in the SZ, vertical alignment in the DZ and more isotropic appearance in the $\mathrm{MZ}$. 


\subsection{Effect of 3DP on cell migration in THA-Col 1 bioink}

3 After characterization of the material microstructure and properties, we investigated the

4 response of hMSCs on aligned anisotropic versus isotropic fibers in the THA-Col 1. The

5 bioink was either printed with a cylindrical needle or extruded from the cartridge without

6 needle attached and compared to casted samples. Live/dead staining of hMSC aggregates

7 embedded in THA-Col 1 (printed versus casted) are shown in Fig. 6 A at day 1 and day 6 .

8 Cells were viable after printing and remained viable over culture time. At day 1, some dead

9 cells (stained in red) were visible mainly in the center of the spheroids. After 6 days, less

10 dead cells were present compared to day 1 in all three groups. The live/dead staining also

11 showed no toxic effect of the addition of $\mathrm{H}_{2} \mathrm{O}_{2}(0.39 \mathrm{mM})$ in THA and THA-Col 1 to initiate

12 enzymatic gelation.

13 During in vitro culture, hMSC migrated from the spheroids in all three groups. Observing the

14 cytoskeleton orientation via phalloidin staining, MSCs alignment was more pronounced in the

15 printed groups, while hMSC showed a more isotropic cytoskeletal orientation in the extruded

16 and casted group. The overlay image of actin filament staining (red), and cell nuclei (blue)

17 demonstrated the unidirectional migration of the cells (Fig. 6 B). 


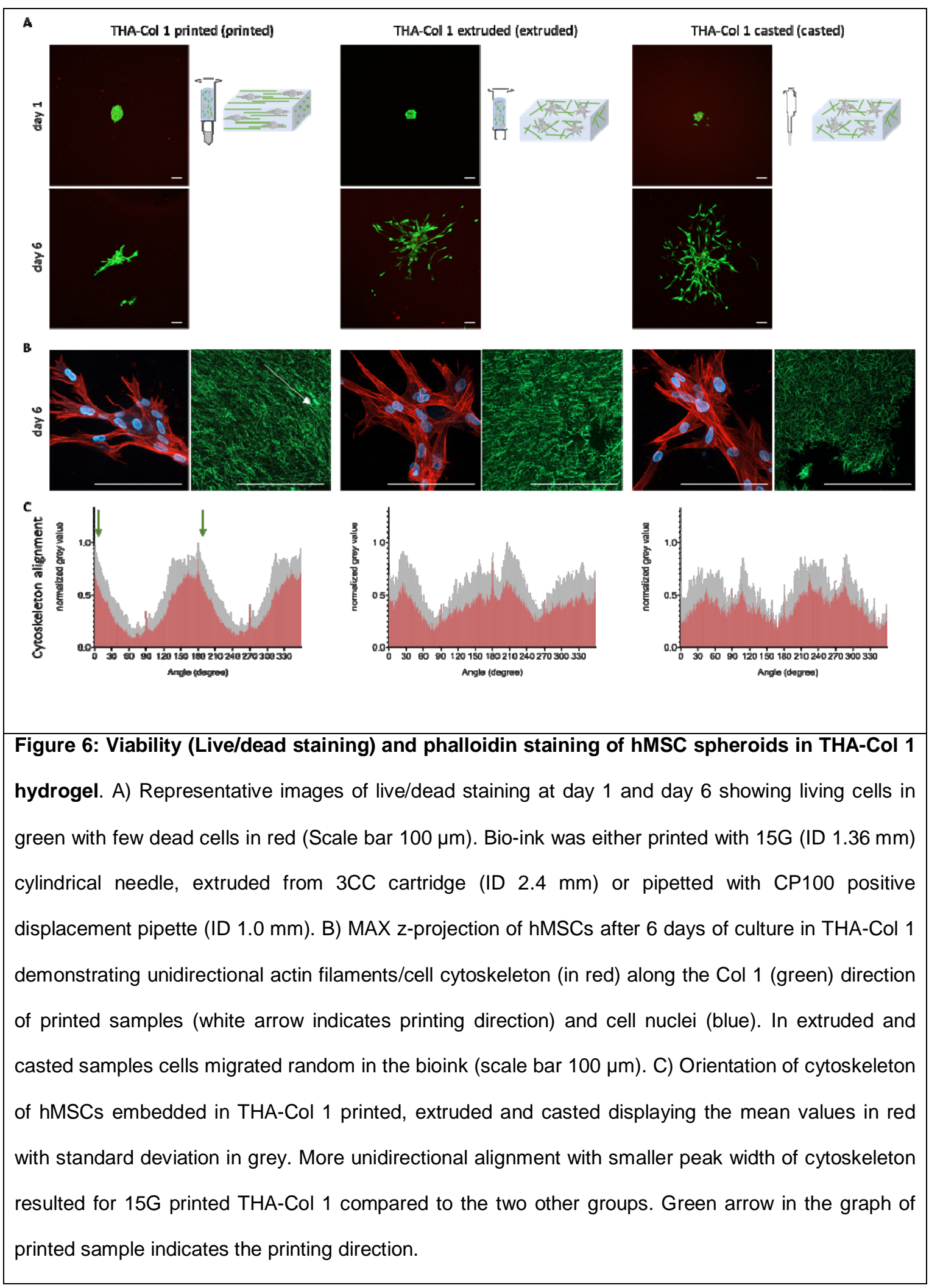


1 Fig. $6 \mathrm{C}$ illustrates the orientation of the cytoskeleton based on MAX projection of the red

2 channel. The group printed with a $15 \mathrm{G}$ needle (left) displays two clear maxima along the

3 orientation of the Col fibers determined by the printing direction (and the $180^{\circ}$ from it),

$4 \quad$ indicated by the green arrows, thus indicating cytoskeleton alignment along the Col fibers.

5 For the group undergoing extrusion without needle $(2.4 \mathrm{~mm}$ diameter, center) the peaks were

6 markedly broadened and more jagged, indicative of a more random distribution; this trend

7 was even more apparent for the casted sample, where the distribution assumes a white

8 noise profile.

\section{Discussion}

11 Extrusion-based printing has achieved significant advances in controlling construct resolution, composition and shape. However, control over the microscopic architecture has been mostly overlooked. Mechanical and biological properties of animal tissues depend not only on the chemical composition, but also on the specific spatial arrangement of structural molecules and biological factors [10]. For example, cartilage is composed of a glycosaminoglycan-based matrix containing Col 1 fibers with specific orientation, parallel to the surface on the superficial zone and perpendicular in deeper layers [31]. In the present work, we propose a technique to introduce anisotropic properties into a THACol 1 composite biomaterial. Having two parallel occurring crosslinking mechanisms, we overcame the difficulty in homogenous mixing Col 1 fibrils into a viscoelastic HA-based matrix by combining a previously developed bioink based on the tyramine derivative of $\mathrm{HA}$ [32] with acidic-solubilized Col 1, which was buffered upon mixing, thereby forming fibrils. Since both precursors, THA bioink and Col 1, are in liquid form, mixing to homogeneity was easily achieved, as visualized with microscopic techniques (Fig. 2 A-D). A distinctive advantage of the preparation method here illustrated is that THA crosslinking and Col 1 fibril formation occur simultaneously, thus avoiding phase separation [23]. Although the Col 1 27 fibrils in the confocal and SHG images in Figure 2 seem to differ between pure Col 1 and 
1 THA-Col 1, both casted, there is no difference in fiber diameter as shown by secondary

2 electron and transmission electron microscopy previously [33]. The visual differences can be

3 explained by the MAX projection of several focus plans within the 3D constructs and thus

4 fibers in the deeper regions tend to display smaller fibrils compared to the fibrils captured in

5 z-directions closer to the objective.

6 The preservation of the fibrillar microstructure of low concentrated neutral Col $1(2.5 \mathrm{mg} / \mathrm{ml}$

7 final concentration in composite) within THA (12.5 mg/ml final concentration) after 3DP is a

8 further feature allowing to produce the anisotropic microstructure.

9 Casted Col 1 hydrogels have been extensively used in the literature, but the fibrillar structure 10 was not extensively investigated when combined with a second polymer. Binner et al. 11 characterized a star shaped poly (ethylene glycol) hydrogel mixed with Fluorescein12 isothiocyanate labelled Col 1. Besides a homogenous distribution of the Col 1, no fibrillar 13 structure was shown and further characterized [34]. Formation of Col 1 fibers resulted to be dependent on the incubation time at $4^{\circ} \mathrm{C}$ prior to $\mathrm{pH}$ neutralization and thus inducing 15 fibrillation when combined with Matrigel [35]. A thermally controlled printing of Col 1 $16(6 \mathrm{mg} / \mathrm{ml})$ blended with Pluronic $(60 \% \mathrm{w} / \mathrm{v})$ resulted in Col 1 fiber formation and aggregation 17 with a dependency of the fiber alignment with the amount of media added and thus time to 18 dissolve the Pluronic out of the blend [21].

19 The shear thinning and viscosity behavior of high concentration neutralized Col 1 (20-60 $\mathrm{mg} / \mathrm{ml}$ ) has been characterized by different groups [20,36]. In contrast, 3D printing of

21 neutralized pure Col 1 at low concentrations is challenging due to the lack of shear thinning 22 and shape retention. At the low concentration used in this study ( $\leq 5 \mathrm{mg} / \mathrm{ml})$, printability was 23 rescued by the shear thinning THA. A similar approach was presented by Duarte Capos et 24 al. using agarose as shear thinning component [37]. On the other hand, printing properties of 25 THA previously investigated in our group were preserved upon mixing with Col 1 [38].

26 The addition of Col 1 to THA increased the storage modulus. Thus, the fibrillar network within 27 the viscoelastic THA can be used as a design parameter to modulate the mechanical 28 properties. The formation of di-tyramine bonds between Col 1 and THA could be one reason 
1 for the synergistic effect $[33,39]$. Other advantages of the composite are that the cell-

2 mediated shrinking of Col 1 scaffolds is reduced; on the other side, Col 1 provides cell

3 attachment sites to the HA, and an ECM-mimetic fibrillar matrix. Reduced shrinkage of Col 1

4 was also observed when this natural material was combined with silk when pulmonary

5 fibroblasts were embedded [39].

6 Within the casted gels the fibrils exhibited either random orientation or local domains with

7 some degree of orientation, which could be attributed to the low but non-zero shear forces

8 experienced during casting from a pipette.

9 After 3D printing of the composite, an overall alignment along the printing axis was observed,

10 as expected. Therefore, the low-grade enzymatic crosslinking of the THA preserved at least

11 in part the capability of the fibrils to comply with the shear stimulus when extruded.

12 Shear-induced alignment of fibrillar structures has been reported by different groups [22, 40].

$13 \mathrm{Kim}$ et al. controlled the fiber alignment in dependence of the needle diameter during printing 14 showing a higher degree of alignment when using 30G compared to 20G needles [40]. This

15 intuitive behavior was not observed in the present study; rather, with thinner needles the 16 fibrillar structure was disrupted, possibly due to the significantly higher pressure that was 17 needed to achieve extrusion, potentially creating discontinuity or turbulence (supplementary 18 Fig. A1). Moncal et al. introduced Col printing $(6 \mathrm{mg} / \mathrm{ml})$ with a thermally controlled set-up using Pluronic as sacrificial material and quantified the alignment of Col fibers along printing direction related to the amount of media and incubation time $(0-48 \mathrm{~h})$ to induce fibrillation but with overall low anisotropy indices [21]. An agarose-Col $(5 \mathrm{mg} / \mathrm{ml}$ agarose, $2 \mathrm{mg} / \mathrm{ml}$ Col 1) blend with equally distributed col fibers but no signs of alignment after printing was presented by Koepf et al. [41]. A different approach to align Col 1 fibers was shown by Betsch et al. They aligned iron nanoparticle loaded agarose-Col 1 biomaterial ink after exposure to a magnetic field. The alignment was most prevalent in Col $1(3 \mathrm{mg} / \mathrm{ml})$ compared to the composite (agarose $3 \mathrm{mg} / \mathrm{ml}$, Col $12.5 \mathrm{mg} / \mathrm{ml}$ ) only in the group with magnetic field exposure

27 [42]. Yang et al. also used electrically assisted printing to mimic Col 1 orientation in meniscus by radial and circumferential aligned carbon nanotubes [43]. With the method here 
1 presented, we achieved alignment of Col 1 fibrils within a composite using exclusively natural

2 ECM molecules avoiding using synthetic non-degradable components simply by 3D

3 extrusion printing. Col 1 only can evolve over time becoming isotropic. This behavior is

4 prevented within the composite, due to the presence of gelled THA stabilizing Col 1 fibrils.

5 The combination of the two ECM components brings biological features and directs cellular

6 behavior. Single cell seeding resulted in enhanced adhesion, overcoming limited cell

7 attachment reported before [38]. Similar results were observed with cell aggregates, where

8 the presence of Col 1 increased the migration length and area. This behavior can be

9 attributed to the presence of the integrin interaction site Arginine, Glycine and Aspartate,

10 (RGD sequence) naturally contained in Col 1 inducing cell adhesion [44]. A direct correlation

11 of cell adhesion and RGD density was shown also for electrospun HA functionalized with

12 RGD peptide by Kim et al. [45]. The above cited agarose-Col blend has been shown to

13 enhance smooth muscle cell spreading and attachment and thus confirming that the

14 presence of Col 1 in a composite influences cell spreading [41].

15 The local microstructure of the material but also fiber parameters regulate cell response [46,

16 47]. In this study, hMSC migration was stimulated along the unidirectional orientation of the

17 Col 1 fibers after 3D printing. Due to the interaction of hMSC with the Col 1 fibers, the original

18 orientation of the parallel fiber alignment was less visible in confocal microscopy (Fig. 6 B).

19 However, the overlapping direction of cytoskeleton orientation and fiber alignment were

20 visualized and quantified (supplementary Fig. A2). In a comparable study, Kim et al. showed

21 the unidirectional Actin filament orientation of keratinocytes embedded in corneal stroma-

22 derived decellularized ECM after printing which were less pronounced in non-printed

23 samples for preparation of corneal implants [40]. Yang et al. reported an increase in tendon

24 associated genes of rat MSC with oriented Col 1 fiber membrane compared to the isotropic

25 sample [48]. Whether this observation relies on the anisotropic properties or is partially

26 induced by the shear stress during printing is not fully understood.

27 HA has been attributed a plethora of biological properties, including inducing cell 28 proliferation, chondrogenic differentiation and matrix synthesis [49, 50]. The THA-Col 1 
1 composite here proposed supported the migration and chondrogenic differentiation of hMSC

2 spheroids, resulting in cartilage like matrix deposition throughout the whole hydrogel. Matrix

3 deposition was uniform, with staining comparable to the standard pellet culture control (Fig. 4

4 E-F). Importantly, despite Col 1 presence and invasion of the whole matrix, cell morphology

5 was also comparable to the pellet culture, showing a more condensed rather than spindle

6 shape morphology. The biomaterial used in this study overcomes the limitation of

7 extracellular matrix deposition only in the pericellular region which is attributed to many

8 seminatural materials used for cartilage tissue engineering.

9 One possible concern regarding cartilage regeneration is the use of Col type 1 instead of

10 type 2 for our bioink. Clinical studies of a cell free type 1 Col hydrogel (CaReS-1S ${ }^{\circledR}$, Arthro

11 Kinetics AG) implanted in focal, full layer cartilage defects have shown good clinical outcome

12 addressing defect filling and homogenous structure of the repair tissue [51], with the Col 1

13 disappearing in vivo to leave space to the cell-deposited Col 2 [52]. In a minipig study

14 colonization of the cell free Col 1 matrix gave outcome comparable to matrix assisted

15 chondrocyte implantation [53, 54]. Jiang et al. demonstrated superior chondrogenic

16 differentiation of MSC embedded in Col 1 compared to MSC only in a rat model [55]. On the

17 other hand, HA has been combined with Col 1 or gelatin before with similar outcomes that

18 chondrogenic differentiation is promoted in the composite compared to HA or Col 1 only [56-

19 58].

20 One limitation of the present technique is the shear-induce alignment, limiting the degrees of

21 spatial freedom in fibril arrangement. Another limitation is the missing quantification of the

22 Col 1 fibers within the composite compared to Col 1 hydrogel. This was due to technical

23 limitations in visualizing Col 1 fibrils crossing different focus planes within a 3D hydrogel and

24 thus dealing with autofluorescence, light scattering and the opaque character of the hydrogel.

25 For further development, the capacity of regenerating cartilage or other tissues should be

26 tested interrogating specifically the effect of the orientation separately from the composition,

27 comparing random oriented or non-fibrillar constructs with aligned constructs. 


\section{Conclusions}

2 Overall, in this work, we have presented a method to obtain an THA-Col 1 composite with

3 macroscopic homogeneity and microscopic heterogeneity mimicking the macromolecular

4 architecture of native tissues. We achieved a uniform distribution of Col 1 fibers within an

5 HA-based viscoelastic matrix starting from liquid precursors with simultaneous Col 1

6 fibrillation and HA crosslinking. The orientation of the Col 1 fibers in the 3D printed construct

7 was controlled with the shear stress during printing and had a direct impact on cell behavior.

8 The biomaterial ink here introduced can be extended to other fibrillar proteins to produce

9 similar microstructural features. The possibility of printing ECM components with control over

10 microscopic alignment brings biofabrication one step closer to capturing the complexity of

11 biological tissues.

Author contribution

Andrea Schwab: Conceptualization, Methodology, Software, Validation, Formal analysis, Investigation, Data Curation, Writing-Original Draft. Christophe Hélary: Conceptualization, Review and Editing. Geoff Richards: Review and Editing, Funding acquisition. Mauro Alini:

17 Conceptualization, Review and Editing, Funding acquisition. David Eglin: Review and Editing, Project administration, Funding acquisition. Matteo D'Este: Conceptualization, Writing-Review and Editing, Supervision, Project administration, Funding acquisition.

\section{Declaration of competing interests}

The authors declare that they have no known competing financial interests or personal relationships that could have appeared to influence the work reported in this paper. 
1 The processed data required to reproduce these findings are available to download from

2 Mendeley-Data link http://dx.doi.org/10.17632/vcxdk7s8jy.2 .

3

4 Acknowledgements

5 This work is part of the osteochondral defect collaborative research program supported by

6 the $\mathrm{AO}$ foundation.

7 This project has been partially supported by "L'Agence Nationale de la Recherche" (ANR)

8 and the Swiss National Science Foundation (SNSF): INDEED project, SNSF's grant number

9 310030E_189310 and ANR's grant number ANR-19-CE06-0028.

10 The authors acknowledge support of the Scientific Center for Optical and Electron

11 Microscopy ScopeM of the Swiss Federal Institute of Technology ETHZ for acquiring SHG

12 images.

13 The Graubünden Innovationsstiftung is acknowledged for its financial support.

14 The authors thank Dr. Christoph Sprecher for his support with confocal microscopy settings

15 and Luca Ambrosio MD for his support setting up the turbidity measurement.

\section{References}

18 [1] S.V. Murphy, A. Atala, 3D bioprinting of tissues and organs, Nat Biotechnol 32(8) (2014)

19 773-85. https://doi.org/10.1038/nbt.2958.

20 [2] M. Hospodiuk, M. Dey, D. Sosnoski, I.T. Ozbolat, The bioink: A comprehensive review on

21 bioprintable materials, Biotechnol Adv 35(2) (2017) 217-239.

22 https://doi.org/10.1016/j.biotechadv.2016.12.006.

23 [3] F.P.W. Melchels, W.J.A. Dhert, D.W. Hutmacher, J. Malda, Development and

24 characterisation of a new bioink for additive tissue manufacturing, J Mater Chem B 2(16)

25 (2014) 2282. https://doi.org/10.1039/c3tb21280g. 
1 [4] J. Malda, J. Visser, F.P. Melchels, T. Jungst, W.E. Hennink, W.J. Dhert, J. Groll, D.W.

2 Hutmacher, 25th anniversary article: Engineering hydrogels for biofabrication, Adv Mater

3 25(36) (2013) 5011-28. https://doi.org/10.1002/adma.201302042.

4 [5] A.K. Miri, I. Mirzaee, S. Hassan, S. Mesbah Oskui, D. Nieto, A. Khademhosseini, Y.S.

5 Zhang, Effective bioprinting resolution in tissue model fabrication, Lab Chip 19(11) (2019)

6 2019-2037. https://doi.org/10.1039/c8lc01037d.

7 [6] S.M. Bittner, B.T. Smith, L. Diaz-Gomez, C.D. Hudgins, A.J. Melchiorri, D.W. Scott, J.P.

8 Fisher, A.G. Mikos, Fabrication and mechanical characterization of 3D printed vertical

9 uniform and gradient scaffolds for bone and osteochondral tissue engineering, Acta Biomater

1090 (2019) 37-48. https://doi.org/10.1016/j.actbio.2019.03.041.

11 [7] T.J. Klein, S.C. Rizzi, J.C. Reichert, N. Georgi, J. Malda, W. Schuurman, R.W. Crawford,

12 D.W. Hutmacher, Strategies for zonal cartilage repair using hydrogels, Macromol Biosci

$139(11)(2009)$ 1049-58. https://doi.org/10.1002/mabi.200900176.

14 [8] L.H. Nguyen, A.K. Kudva, N.S. Saxena, K. Roy, Engineering articular cartilage with

15 spatially-varying matrix composition and mechanical properties from a single stem cell

16 population using a multi-layered hydrogel, Biomaterials 32(29) (2011) 6946-52.

17 https://doi.org/10.1016/j.biomaterials.2011.06.014.

18 [9] M.A. Heinrich, W. Liu, A. Jimenez, J. Yang, A. Akpek, X. Liu, Q. Pi, X. Mu, N. Hu, R.M.

19 Schiffelers, J. Prakash, J. Xie, Y.S. Zhang, 3D Bioprinting: from Benches to Translational

20 Applications, Small 15(23) (2019) e1805510. https://doi.org/10.1002/smll.201805510.

21 [10] P. Datta, V. Vyas, S. Dhara, A.R. Chowdhury, A. Barui, Anisotropy Properties of

22 Tissues: A Basis for Fabrication of Biomimetic Anisotropic Scaffolds for Tissue Engineering,

23 Journal of Bionic Engineering 16(5) (2019) 842-868. https://doi.org/10.1007/s42235-019-

$24 \quad 0101-9$.

25 [11] M.D. Shoulders, R.T. Raines, Collagen structure and stability, Annu Rev Biochem 78

26 (2009) 929-58. https://doi.org/10.1146/annurev.biochem.77.032207.120833. 
1 [12] A. Malandrino, X. Trepat, R.D. Kamm, M. Mak, Dynamic filopodial forces induce

2 accumulation, damage, and plastic remodeling of 3D extracellular matrices, PLoS Comput

3 Biol 15(4) (2019) e1006684. https://doi.org/10.1371/journal.pcbi.1006684.

4 [13] A.D. Doyle, K.M. Yamada, Mechanosensing via cell-matrix adhesions in 3D

5 microenvironments, Exp Cell Res 343(1) (2016) 60-66.

6 https://doi.org/10.1016/j.yexcr.2015.10.033.

7 [14] W.M. Han, S.J. Heo, T.P. Driscoll, J.F. Delucca, C.M. McLeod, L.J. Smith, R.L. Duncan,

8 R.L. Mauck, D.M. Elliott, Microstructural heterogeneity directs micromechanics and

9 mechanobiology in native and engineered fibrocartilage, Nat Mater 15(4) (2016) 477-84.

10 https://doi.org/10.1038/nmat4520.

11 [15] B. Patel, Z. Xu, C.B. Pinnock, L.S. Kabbani, M.T. Lam, Self-assembled Collagen-Fibrin

12 Hydrogel Reinforces Tissue Engineered Adventitia Vessels Seeded with Human Fibroblasts,

13 Sci Rep 8(1) (2018) 3294. https://doi.org/10.1038/s41598-018-21681-7.

14 [16] A.S. Gladman, E.A. Matsumoto, R.G. Nuzzo, L. Mahadevan, J.A. Lewis, Biomimetic 4D 15 printing, Nat Mater 15(4) (2016) 413-8. https://doi.org/10.1038/nmat4544.

16 [17] S.A. Bradner, M. McGill, A. Golding, R. Grudt, D.L. Kaplan, Silk Hydrogel Microfibers for 17 Biomimetic Fibrous Material Design, Macromol Mat Eng 304(7) (2019) 1900045.

18 https://doi.org/10.1002/mame.201900045.

19 [18] A. Lode, M. Meyer, S. Bruggemeier, B. Paul, H. Baltzer, M. Schropfer, C. Winkelmann, 20 F. Sonntag, M. Gelinsky, Additive manufacturing of collagen scaffolds by three-dimensional 21 plotting of highly viscous dispersions, Biofabrication 8(1) (2016) 015015.

22 https://doi.org/10.1088/1758-5090/8/1/015015.

23 [19] N. Diamantides, C. Dugopolski, E. Blahut, S. Kennedy, L.J. Bonassar, High density cell 24 seeding affects the rheology and printability of collagen bioinks, Biofabrication 11(4) (2019) 25 045016. https://doi.org/10.1088/1758-5090/ab3524.

26 [20] E.O. Osidak, P.A. Karalkin, M.S. Osidak, V.A. Parfenov, D.E. Sivogrivov, F. Pereira, A.A.

27 Gryadunova, E.V. Koudan, Y.D. Khesuani, C.V.A. capital Ka, S.I. Belousov, S.V.

28 Krasheninnikov, T.E. Grigoriev, S.N. Chvalun, E.A. Bulanova, V.A. Mironov, S.P. 
1 Domogatsky, Viscoll collagen solution as a novel bioink for direct 3D bioprinting, J Mater Sci

2 Mater Med 30(3) (2019) 31. https://doi.org/10.1007/s10856-019-6233-y.

3 [21] K.K. Moncal, V. Ozbolat, P. Datta, D.N. Heo, I.T. Ozbolat, Thermally-controlled

4 extrusion-based bioprinting of collagen, J Mater Sci Mater Med 30(5) (2019) 55.

5 https://doi.org/10.1007/s10856-019-6258-2.

6 [22] B.A. Nerger, P.T. Brun, C.M. Nelson, Microextrusion printing cell-laden networks of type

7 I collagen with patterned fiber alignment and geometry, Soft Matter 15(28) (2019) 5728-5738.

8 https://doi.org/10.1039/c8sm02605j.

9 [23] S. Rhee, J.L. Puetzer, B.N. Mason, C.A. Reinhart-King, L.J. Bonassar, 3D Bioprinting of 10 Spatially Heterogeneous Collagen Constructs for Cartilage Tissue Engineering, ACS

11 Biomater Sci Eng 2(10) (2016) 1800-1805. https://doi.org/10.1021/acsbiomaterials.6b00288.

12 [24] R.V. lozzo, L. Schaefer, Proteoglycan form and function: A comprehensive

13 nomenclature of proteoglycans, Matrix Biol 42 (2015) 11-55.

14 https://doi.org/10.1016/j.matbio.2015.02.003.

15 [25] C. Loebel, M. D'Este, M. Alini, M. Zenobi-Wong, D. Eglin, Precise tailoring of tyramine-

16 based hyaluronan hydrogel properties using DMTMM conjugation, Carbohydr Polym 115

17 (2015) 325-33. https://doi.org/10.1016/j.carbpol.2014.08.097.

18 [26] O.F. Gardner, M. Alini, M.J. Stoddart, Mesenchymal Stem Cells Derived from Human

19 Bone Marrow, Methods Mol Biol 1340 (2015) 41-52. https://doi.org/10.1007/978-1-4939-

20 2938-2_3.

21 [27] C. Loebel, T. Stauber, M. D'Este, M. Alini, M. Zenobi-Wong, D. Eglin, Fabrication of cell-

22 compatible hyaluronan hydrogels with a wide range of biophysical properties through high

23 tyramine functionalization, J Mater Chem B 5(12) (2017) 2355-2363.

24 https://doi.org/10.1039/c6tb03161g.

25 [28] R. Tognato, A.R. Armiento, V. Bonfrate, R. Levato, J. Malda, M. Alini, D. Eglin, G.

26 Giancane, T. Serra, A Stimuli-Responsive Nanocomposite for 3D Anisotropic Cell-Guidance

27 and Magnetic Soft Robotics, Adv Funct Mater 29(9) (2019) 1804647.

28 https://doi.org/10.1002/adfm.201804647. 
1 [29] C.E. Ayres, B.S. Jha, H. Meredith, J.R. Bowman, G.L. Bowlin, S.C. Henderson, D.G.

2 Simpson, Measuring fiber alignment in electrospun scaffolds: a user's guide to the 2D fast

3 Fourier transform approach, J Biomater Sci Polym Ed 19(5) (2008) 603-21.

4 https://doi.org/10.1163/156856208784089643.

5 [30] R. Farndale, D. Buttle, A. Barrett, Improved quantitation and discrimination of sulphated

6 glycosaminoglycans by use of dimethylmethylene blue, Biochim Biophys Acta Gen Subj

7 883(2) (1986) 173-177. https://doi.org/10.1016/0304-4165(86)90306-5.

8 [31] J.A. Buckwalter, V.C. Mow, A. Ratcliffe, Restoration of Injured or Degenerated Articular

9 Cartilage, J Am Acad Orthop Surg 2(4) (1994) 192-201. https://doi.org/10.5435/00124635-

$10 \quad 199407000-00002$.

11 [32] D. Petta, A.R. Armiento, D. Grijpma, M. Alini, D. Eglin, M. D'Este, 3D bioprinting of a

12 hyaluronan bioink through enzymatic-and visible light-crosslinking, Biofabrication 10(4)

13 (2018) 044104. https://doi.org/10.1088/1758-5090/aadf58.

14 [33] A. Frayssinet, D. Petta, C. Illoul, B. Haye, A. Markitantova, D. Eglin, G. Mosser, M.

15 D'Este, C. Helary, Extracellular matrix-mimetic composite hydrogels of cross-linked

16 hyaluronan and fibrillar collagen with tunable properties and ultrastructure, Carbohydr Polym

17236 (2020) 116042. https://doi.org/10.1016/j.carbpol.2020.116042.

18 [34] M. Binner, L.J. Bray, J. Friedrichs, U. Freudenberg, M.V. Tsurkan, C. Werner, Cell-

19 instructive starPEG-heparin-collagen composite matrices, Acta Biomater 53 (2017) 70-80.

20 https://doi.org/10.1016/j.actbio.2017.01.086.

21 [35] K.V. Nguyen-Ngoc, A.J. Ewald, Mammary ductal elongation and myoepithelial migration

22 are regulated by the composition of the extracellular matrix, J Microsc 251 (3) (2013) 212-23.

23 https://doi.org/10.1111/jmi.12017.

24 [36] A.D. Nocera, R. Comin, N.A. Salvatierra, M.P. Cid, Development of 3D printed fibrillar

25 collagen scaffold for tissue engineering, Biomed Microdevices 20(2) (2018) 26.

26 https://doi.org/10.1007/s10544-018-0270-z.

27 [37] D.F. Duarte Campos, M. Rohde, M. Ross, P. Anvari, A. Blaeser, M. Vogt, C. Panfil, G.H.

28 Yam, J.S. Mehta, H. Fischer, P. Walter, M. Fuest, Corneal bioprinting utilizing collagen- 
1 based bioinks and primary human keratocytes, J Biomed Mater Res A 107(9) (2019) 1945-

2 1953. https://doi.org/10.1002/jbm.a.36702.

3 [38] D. Petta, D.W. Grijpma, M. Alini, D. Eglin, M. D’Este, Three-Dimensional Printing of a

4 Tyramine Hyaluronan Derivative with Double Gelation Mechanism for Independent Tuning of

5 Shear Thinning and Postprinting Curing, ACS Biomater Sci Eng 4(8) (2018) 3088-3098.

6 https://doi.org/10.1021/acsbiomaterials.8b00416.

7 [39] A. Sundarakrishnan, H. Zukas, J. Coburn, B.T. Bertini, Z. Liu, I. Georgakoudi, L. Baugh,

8 Q. Dasgupta, L.D. Black, D.L. Kaplan, Bioengineered in Vitro Tissue Model of Fibroblast

9 Activation for Modeling Pulmonary Fibrosis, ACS Biomater Sci Eng 5(5) (2019) 2417-2429.

10 https://doi.org/10.1021/acsbiomaterials.8b01262.

11 [40] H. Kim, J. Jang, J. Park, K.P. Lee, S. Lee, D.M. Lee, K.H. Kim, H.K. Kim, D.W. Cho,

12 Shear-induced alignment of collagen fibrils using 3D cell printing for corneal stroma tissue engineering, Biofabrication 11(3) (2019) 035017. https://doi.org/10.1088/1758-5090/ab1a8b.

14 [41] M. Koepf, D.F. Campos, A. Blaeser, K.S. Sen, H. Fischer, A tailored three-dimensionally 15 printable agarose-collagen blend allows encapsulation, spreading, and attachment of human 16 umbilical artery smooth muscle cells, Biofabrication 8(2) (2016) 025011.

17 https://doi.org/10.1088/1758-5090/8/2/025011.

18 [42] M. Betsch, C. Cristian, Y.Y. Lin, A. Blaeser, J. Schoneberg, M. Vogt, E.M. Buhl, H. 19 Fischer, D.F. Duarte Campos, Incorporating 4D into Bioprinting: Real-Time Magnetically Directed Collagen Fiber Alignment for Generating Complex Multilayered Tissues, Adv 21 Healthc Mater 7(21) (2018) e1800894. https://doi.org/10.1002/adhm.201800894.

22 [43] Y. Yang, Z. Chen, X. Song, Z. Zhang, J. Zhang, K.K. Shung, Q. Zhou, Y. Chen, 23 Biomimetic Anisotropic Reinforcement Architectures by Electrically Assisted Nanocomposite 24 3D Printing, Adv Mater 29(11) (2017) https://doi.org/10.1002/adma.201605750.

25 [44] M. Baniasadi, M. Minary-Jolandan, Alginate-Collagen Fibril Composite Hydrogel, 26 Materials (Basel) 8(2) (2015) 799-814. https://doi.org/10.3390/ma8020799. 
1 [45] I.L. Kim, S. Khetan, B.M. Baker, C.S. Chen, J.A. Burdick, Fibrous hyaluronic acid

2 hydrogels that direct MSC chondrogenesis through mechanical and adhesive cues,

3 Biomaterials 34(22) (2013) 5571-80. https://doi.org/10.1016/j.biomaterials.2013.04.004.

4 [46] A.D. Doyle, N. Carvajal, A. Jin, K. Matsumoto, K.M. Yamada, Local 3D matrix

5 microenvironment regulates cell migration through spatiotemporal dynamics of contractility-

6 dependent adhesions, Nat Commun 6 (2015) 8720. https://doi.org/10.1038/ncomms9720.

7 [47] T.L. Jenkins, D. Little, Synthetic scaffolds for musculoskeletal tissue engineering: cellular

8 responses to fiber parameters, NPJ Regen Med 4 (2019) 15. https://doi.org/10.1038/s41536-

$9 \quad 019-0076-5$.

10 [48] S. Yang, X. Shi, X. Li, J. Wang, Y. Wang, Y. Luo, Oriented collagen fiber membranes

11 formed through counter-rotating extrusion and their application in tendon regeneration,

12 Biomaterials 207 (2019) 61-75. https://doi.org/10.1016/j.biomaterials.2019.03.041.

13 [49] R. Mohan, N. Mohan, D. Vaikkath, Hyaluronic Acid Dictates Chondrocyte Morphology

14 and Migration in Composite Gels, Tissue Eng Part A 24(19-20) (2018) 1481-1491.

15 https://doi.org/10.1089/ten.TEA.2017.0411.

16 [50] E. Amann, P. Wolff, E. Breel, M. van Griensven, E.R. Balmayor, Hyaluronic acid

17 facilitates chondrogenesis and matrix deposition of human adipose derived mesenchymal

18 stem cells and human chondrocytes co-cultures, Acta Biomater 52 (2017) 130-144.

19 https://doi.org/10.1016/j.actbio.2017.01.064.

20 [51] T. Efe, C. Theisen, S. Fuchs-Winkelmann, T. Stein, A. Getgood, M.B. Rominger, J.R.

21 Paletta, M.D. Schofer, Cell-free collagen type I matrix for repair of cartilage defects-clinical

22 and magnetic resonance imaging results, Knee Surg Sports Traumatol Arthrosc 20(10)

23 (2012) 1915-22. https://doi.org/10.1007/s00167-011-1777-5.

24 [52] K.F. Schuettler, J. Struewer, M.B. Rominger, P. Rexin, T. Efe, Repair of a chondral

25 defect using a cell free scaffold in a young patient--a case report of successful scaffold

26 transformation and colonisation, BMC Surg 13 (2013) 11. https://doi.org/10.1186/1471-2482-

$27 \quad 13-11$. 
1 [53] K. Gavenis, U. Schneider, U. Maus, T. Mumme, R. Muller-Rath, B. Schmidt-Rohlfing, S.

2 Andereya, Cell-free repair of small cartilage defects in the Goettinger minipig: which defect

3 size is possible?, Knee Surg Sports Traumatol Arthrosc 20(11) (2012) 2307-14.

4 https://doi.org/10.1007/s00167-011-1847-8.

5 [54] U. Schneider, B. Schmidt-Rohlfing, K. Gavenis, U. Maus, R. Mueller-Rath, S. Andereya,

6 A comparative study of 3 different cartilage repair techniques, Knee Surg Sports Traumatol

7 Arthrosc 19(12) (2011) 2145-52. https://doi.org/10.1007/s00167-011-1460-x.

8 [55] X. Jiang, X. Huang, T. Jiang, L. Zheng, J. Zhao, X. Zhang, The role of Sox9 in collagen

9 hydrogel-mediated chondrogenic differentiation of adult mesenchymal stem cells (MSCs),

10 Biomater Sci 6(6) (2018) 1556-1568. https://doi.org/10.1039/c8bm00317c.

11 [56] C.G. Pfeifer, A. Berner, M. Koch, W. Krutsch, R. Kujat, P. Angele, M. Nerlich, J. Zellner,

12 Higher Ratios of Hyaluronic Acid Enhance Chondrogenic Differentiation of Human MSCs in a

13 Hyaluronic Acid-Gelatin Composite Scaffold, Materials (Basel) 9(5) (2016)

14 https://doi.org/10.3390/ma9050381.

15 [57] V. Moulisova, S. Poveda-Reyes, E. Sanmartin-Masia, L. Quintanilla-Sierra, M.

16 Salmeron-Sanchez, G. Gallego Ferrer, Hybrid Protein-Glycosaminoglycan Hydrogels

17 Promote Chondrogenic Stem Cell Differentiation, ACS Omega 2(11) (2017) 7609-7620.

18 https://doi.org/10.1021/acsomega.7b01303.

19 [58] J. Yang, Y. Liu, L. He, Q. Wang, L. Wang, T. Yuan, Y. Xiao, Y. Fan, X. Zhang, Icariin

20 conjugated hyaluronic acid/collagen hydrogel for osteochondral interface restoration, Acta

21 Biomater 74 (2018) 156-167. https://doi.org/10.1016/j.actbio.2018.05.005. 


\section{Figure captions}

2 Figure 7: 3D bioprinting as a tool to produce microscopic anisotropic scaffolds. Biomaterial and

3 bioink were prepared by mixing neutralized Col $1(5 \mathrm{mg} / \mathrm{ml})$ isolated from rat tails with tyramine 4 modified HA (THA, $25 \mathrm{mg} / \mathrm{ml})$. Neutralized Col 1 was mixed with THA for enzymatic crosslinking either 5 cell free or containing hMSC cell spheroids. The Col 1 microstructure was investigated after 3D 6 printing and compared to casted, isotropic samples with different microscopic techniques (Second 7 Harmonic Generation SHG imaging and confocal microscopy). Cell instructive properties were analyzed after in vitro culture on cell migration and attachment.

Figure 8: Collagen (Col 1) fibrils distribution in THA hydrogel and rheological properties. A)

Visualization of Col 1 fibrils within Col 1 hydrogel and B) THA-Col 1 hydrogels using confocal imaging (immunofluorescent staining for Col 1) and C-D) SHG imaging (no labelling), respectively. Both techniques illustrate homogenous distribution of Col 1 fibrils (scale bar $100 \mu \mathrm{m}$ ). E) Turbidity measurement of THA-Col 1 demonstrated Col 1 fibril formation in composite dependent on Col 1 content. The more Col 1 is present in composite the higher the absorbance at $313 \mathrm{~nm}$ over time with maximum values for Col 1 only. F) Flow curve (viscosity as function of shear rate) demonstrate shear thinning behavior of THA, THA-Col 1 and neutralized Col 1 marked by decreasing viscosity with increasing shear rate. Col $1(\mathrm{pH} 7,5 \mathrm{mg} / \mathrm{ml})$ showed lower viscosity compared to THA (25 mg/ml) and THA-Col 1. Acidic Col $1(\mathrm{pH} 4,5 \mathrm{mg} / \mathrm{ml})$ lacks shear-thinning behavior with a constant viscosity over range of tested shear rate. G) Rheological characterization (Amplitude sweep, $1 \mathrm{~Hz}$ ) to evaluate mechanical properties of biomaterial ink by means of storage ( $\left.G^{\prime}\right)$ and loss $\left(G^{\prime \prime}\right)$ modulus of THA-Col 1 and THA after enzymatic crosslinking. Storage modulus increases in composite comparted to THA.

Figure 9: Cell instructive properties of THA-Col 1 addressing hMSC migration and cell attachment. A) Confocal images of actin filament stained hMSC spheroids embedded in THA-Col 1 ( THA-Col 1, $25 \mathrm{mg} / \mathrm{ml} 1: 1 \mathrm{Col} 15 \mathrm{mg} / \mathrm{ml})$, THA (25 mg/ml) and Col $1(5 \mathrm{mg} / \mathrm{ml})$ at day 0 , day 3 and after 8 days culture. Migration of hMSC was seen for THA-Col 1 and Col 1 but not in THA. Col 1 hydrogel was shrinking over time and formed a small pellet of less than $2 \mathrm{~mm}$ in diameter (Scale bar $100 \mu \mathrm{m})$. B) Area of cell migration and C) migration length was higher in Col 1 compared to THA-Col 1 increasing over time. Quantification of migration area and migration length. ${ }^{* *} p<0.01,{ }^{* * *} p<0.001$. 
2 Figure 10: Chondrogenic differentiation potential of hMSC embedded in THA-Col 1 compared to

3 hMSC pellet culture. A) Gene expression analysis relative to hMSC pellet or hMSC embedded in 4 THA-Col 1 hydrogel (TC MSC) control at day 0 for Col 1 (COL 1A1), Col II (COL 2A1), aggrecan

5 (ACAN), SOX9 and RunX2. Increase in all chondrogenic related genes with minor changes for SOX9 6 and RunnX2. B) Relative gene expression ratio of Col 2 to Col 1 at day 21. No statistical differences 7 resulted for all PCR data shown beside for Col2/Col 1 ratio. $\left.{ }^{*} p<0.05 \mathrm{C}\right)$ Quantification of proteoglycans 8 (GAG/sample) including total amount of GAGs released into the media supernatant and GAGs 9 retained in the sample at day 21. D) GAG/DNA increased over time from day 0 to day 21 for MSC pellet and THA-Col 1 MSC. ${ }^{* * *} p<0.0001$. E) Safranin $O$ staining to visualize proteoglycans in $E C M$ after 21 days in vitro culture at two magnifications for MSC pellet and F) MSC embedded in THA-Col 1 hydrogel (THA-Col 1 MSC) (scale bar $100 \mu \mathrm{m}$ ).

Figure 11: 3DP as tool to align Col 1 fibers and produce microscopically anisotropic scaffolds.

A) Confocal microscopy of immunofluorescent staining against Col 1 and C) SHG images demonstrate anisotropic fibers in THA-Col 1 biomaterial ink (scale bar $100 \mu \mathrm{m}$ ). Unidirectional orientation of parallel Col 1 fibers aligning along the printing direction (white arrow indicated printing direction). B, D) Bar diagrams (mean values \pm standard deviation) display the quantification of fiber alignment in casted Col 1, casted THA-Col 1 (ID $1.0 \mathrm{~mm}$ ) and printed THA-Col 1 (15G: ID $1.36 \mathrm{~mm}, 0.25 "$ cylindrical needle) from images acquired either with confocal microscope (B, green) or SHG (D, blue). Independently of microscopical method the printed THA-Col 1 samples resulted in unidirectional orientation which was more random for casted THA-Col 1 and Col 1 . White arrow in the bar diagram indicate the peak width at a normalized grey value of 0.5 . E) Schematic illustration of Col 1 fiber alignment in articular cartilage. F) Polarized light image of articular cartilage illustrating horizontal fibers in superficial zone (SZ) and vertical orientation in deep zone (DZ) (scale bar $50 \mu \mathrm{m})$. G) Confocal images of immunefluorescent labelled Col 1 mimicking hierarchical fiber orientation in articular cartilage with parallel horizonal fibers in the SZ, more isotropic fibers in the middle zone (MZ) and parallel vertical fibers in the DZ (scale bar $50 \mu \mathrm{m}$ ). 


\section{Figure 12: Viability (Live/dead staining) and phalloidin staining of hMSC spheroids in THA-Col}

21 hydrogel. A) Representative images of live/dead staining at day 1 and day 6 showing living cells in 3 green with few dead cells in red (Scale bar $100 \mu \mathrm{m}$ ). Bio-ink was either printed with 15G (ID $1.36 \mathrm{~mm}$ ) 4 cylindrical needle, extruded from 3CC cartridge (ID $2.4 \mathrm{~mm}$ ) or pipetted with CP100 positive 5 displacement pipette (ID $1.0 \mathrm{~mm}$ ). B) MAX z-projection of hMSCs after 6 days of culture in THA-Col 1 6 demonstrating unidirectional actin filaments/cell cytoskeleton (in red) along the Col 1 (green) direction 7 of printed samples (white arrow indicates printing direction) and cell nuclei (blue). In extruded and 8 casted samples cells migrated random in the bioink (scale bar $100 \mu \mathrm{m}$ ). C) Orientation of cytoskeleton 9 of hMSCs embedded in THA-Col 1 printed, extruded and casted displaying the mean values in red 10 with standard deviation in grey. More unidirectional alignment with smaller peak width of cytoskeleton 11 resulted for $15 \mathrm{G}$ printed THA-Col 1 compared to the two other groups. Green arrow in the graph of 12 printed sample indicates the printing direction. 


\section{Appendix A. Supplementary information}

\section{A.1 Supplementary Materials and Methods}

$3 \quad$ A.1.1 3DP THA-Col 1 biomaterial ink with different needle sizes

4 To compare the influence of needle diameter on Col 1 fiber alignment, THA-Col 1 was 5 extruded (3D Discovery ${ }^{\mathrm{TM}}$, RegenHU) through a $15 \mathrm{G}$ and $22 \mathrm{G}$ needle $(0.25$ " cylindrical 6 needles, 15G: ID $1.36 \mathrm{~mm}, 0.2$ bar, feed rate $8 \mathrm{~mm} / \mathrm{s}$; 22G: ID $0.41 \mathrm{~mm}, 1.5$ bar, feed rate

$78 \mathrm{~mm} / \mathrm{s}$, Nordson EFD) with subsequent light crosslinking (515 nm LED, feed rate $4 \mathrm{~mm} / \mathrm{s}$ ).

8 THA-Col 1 (1:1) biomaterial ink was transferred into 3CC barred (ID $2.3 \mathrm{~mm}$, Nordson) for 9 enzymatic crosslinking $\left(30 \mathrm{~min}, 37^{\circ} \mathrm{C}\right)$, printed with the above-mentioned parameters and 10 light crosslinked. Col 1 fibers were visualized by fluorescent microscopy after staining as 11 described in 2.8.1. To quantify fiber orientation the Oval Plugin (imageJ, NIH) was done similar as for samples described in 2.10.

Table A1: Primer and probe (Forward, reverse and probe sequence) and Assay-On-Demand

(Applied biosystem Assay ID) for gene expression analysis.

\begin{tabular}{|l|l|l|l|l|}
\hline gene & Forward primer & Reverse primer & Probe sequence & Assay ID \\
sequence & sequence & & \\
\hline hACAN & 5'-AGT CCT CAA & 5'-CGG GAA GTG & 5'-CCG GAA TGG AAA & - \\
GCC TCC TGT & GCG GTA ACA-3' & CGT GAA TCA GAA & \\
ACT CA-3' & TCA ACT-3' & & \\
\hline
\end{tabular}




\begin{tabular}{|c|c|c|c|c|}
\hline hCOL1A1 & $\begin{array}{l}\text { 5'-CCC TGG AAA } \\
\text { GAA TGG AGA } \\
\text { TGA T-3' }\end{array}$ & $\begin{array}{c}\text { 5'-ACT GAA ACC } \\
\text { TCT GTG TCC CTT } \\
\text { CA-3' }\end{array}$ & $\begin{array}{l}\text { 5'-CGG GCA ATC CTC } \\
\text { GAG CAC CCT -3' }\end{array}$ & - \\
\hline hCOL2A1 & $\begin{array}{l}\text { 5'-GGC AAT AGC } \\
\text { AGG TTC ACG } \\
\text { TAC A-3' }\end{array}$ & $\begin{array}{l}\text { 5'-GAT AAC AGT } \\
\text { CTT GCC CCA CTT } \\
\text { ACC-3' }\end{array}$ & $\begin{array}{l}\text { 5'-CCT GAA GGA TGG } \\
\text { CTG CAC GAA ACA } \\
\text { TAC-3' }\end{array}$ & - \\
\hline $\begin{array}{l}\text { hCOL10 } \\
\text { A1 }\end{array}$ & $\begin{array}{l}\text { 5'-ACG CTG AAC } \\
\text { GAT ACC AAA } \\
\text { TG-3' }\end{array}$ & $\begin{array}{l}\text { 5'-TGC TAT ACC } \\
\text { TTT ACT CTT TAT } \\
\text { GGT GTA-3' }\end{array}$ & $\begin{array}{l}\text { 5'-ACT ACC CAA CAC } \\
\text { CAA GAC ACA GTT } \\
\text { СTT САT TCC-3' }\end{array}$ & - \\
\hline hRUNX2 & $\begin{array}{l}\text { 5'-AGC AAG GTT } \\
\text { CAA CGA TCT } \\
\text { GAG AT-3' }\end{array}$ & $\begin{array}{l}\text { 5'-TTT GTG AAG } \\
\text { ACG GTT ATG GTC } \\
\text { AA-3' }\end{array}$ & $\begin{array}{l}\text { 5'-TGA AAC TCT TGC } \\
\text { CTC GTC CAC TCC G- } \\
3^{\prime}\end{array}$ & - \\
\hline hUBC & & & & Hs00824723_m1 \\
\hline hSOX9 & & & & Hs00165814_m1 \\
\hline
\end{tabular}

2 A.2 Supplementary Results

3 A.2.1 3DP THA-Col 1 biomaterial ink with different needle sizes 

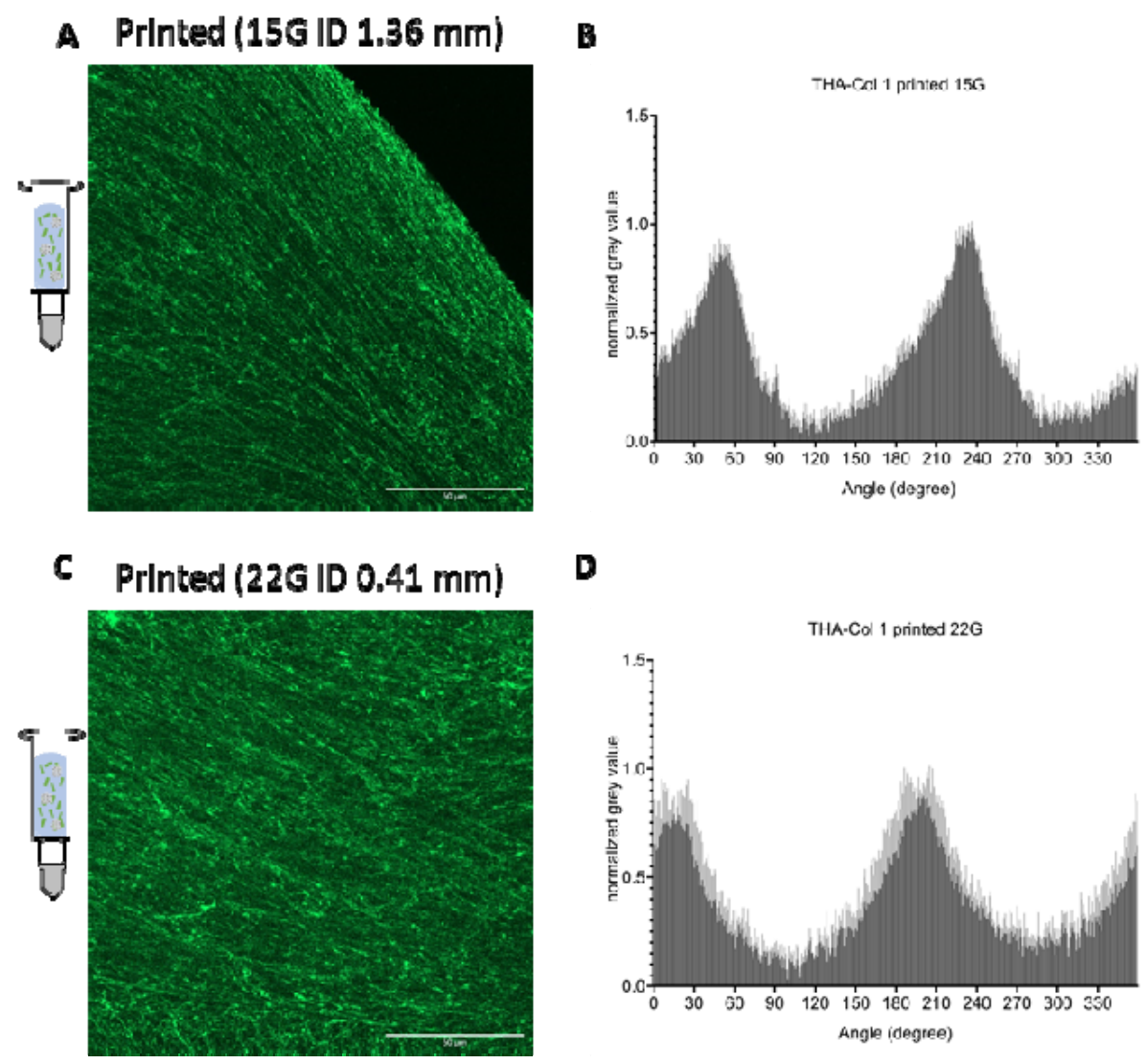

\section{D}

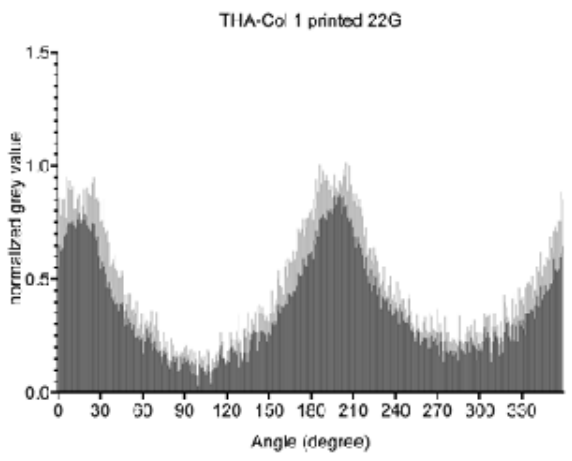

2 Figure A1: Immunofluorescent staining for collagen I of printed THA-Col 1 with 15G (left) and

$322 G$ (right) needle including printing parameters. A) Orientation of fibers did not display distinct

4 differences. With the thinner needle diameter (22G, ID $0.41 \mathrm{~mm}$ ) the fibers appear to become

5 disrupted compared to the bigger needle diameter (15G, ID 1.36 mm). Scale bar 50um. B) Bar

6 diagram displaying the quantification of fiber orientation in printed THA-Col 1 based on immune-

7 fluorescent stained images. Peak width at normalized grey value 0.5 was smaller for $15 G$ needle (51

8 degrees) compared to 22G needle (55 degrees) indicating a more heterogenous fiber orientation for

9 the $22 G$ needle. 
1 A.2.2 3DP hMSC embedded in THA-Col 1 to analyze orientation of cell

2 migration

3 Overlay of results from orientation of hMSC cytoskeleton and Col 1 fibers as described in

42.10 to illustrate the unidirectional migration of hMSC along the direction of Col 1 fibers.

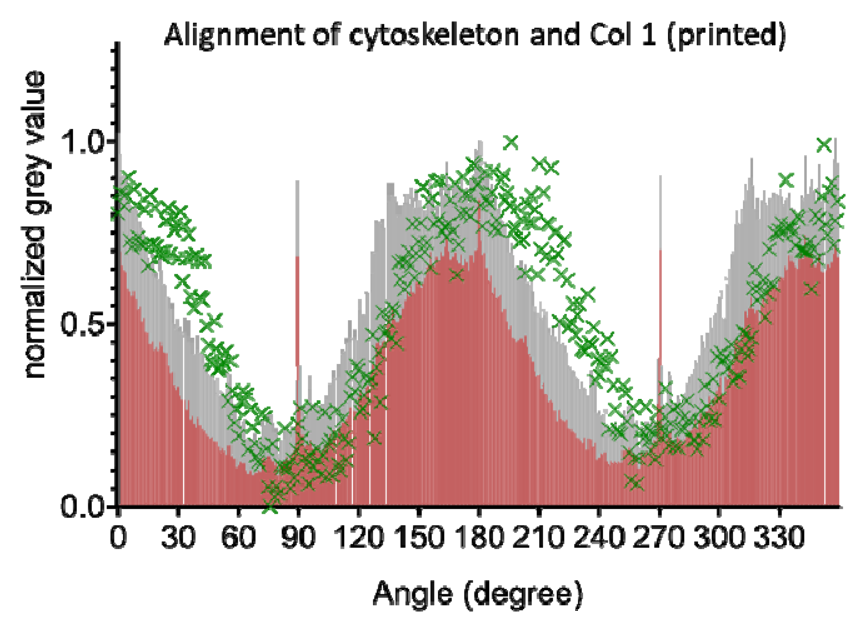

6 Figure A2: Alignment of cytoskeleton and Col 1 fibrils in printed (15G) THA-Col 1 at day 6.

7 Overlay of mean orientation of cytoskeleton (red) with standard deviation in grey and the Col 1

8 orientation (green) to demonstrate the unidirectional migration of hMSCs preferably along the the

9 direction of Col 1 fibers (grey). 